\title{
COMPUTING SPECIAL SMARANDACHE CURVES ACCORDING TO DARBOUX FRAME IN EUCLIDEAN 4-SPACE
}

\author{
M. KHALIFA SAAD ${ }^{1,2, *}$ AND M. A. ABD-RABO ${ }^{3}$ \\ ${ }^{1}$ Department of Mathematics, Faculty of Science, Islamic University of Madinah, 170 Madinah, KSA \\ ${ }^{2}$ Department of Mathematics, Faculty of Science, Sohag University, 82524 Sohag, Egypt \\ ${ }^{3}$ School of Mathematics and Statistics, Zhengzhou University, 450001 Zhengzhou, China \\ *Corresponding author: m_khalifag@yahoo.com, mohammed.khalifa@iu.edu.sa
}

\begin{abstract}
In this paper, we study some special Smarandache curves and their differential geometric properties according to Darboux frame in Euclidean 4-space $\mathbb{E}^{4}$. Also, we compute some of these curves which lie fully on a hypersurface in $\mathbb{E}^{4}$. Moreover, we defray some computational examples in support our main results.
\end{abstract}

\section{INTRODUCTION}

The geometric modeling of free-form curves and surfaces is of central importance for sophisticated CAD/CAM systems. Among all space curves, Smarandache curves have special emplacement regarding their properties, because of this, they deserve especial attention in Euclidean geometry as well as in other geometries. It is known that Smarandache geometry is a geometry which has at least one Smarandache denied axiom [1]. An axiom is said to be Smarandache denied, if it behaves in at least two different ways within the same space. Smarandache geometries are connected with the theory of relativity and the parallel universes. Smarandache curves are the objects of Smarandache geometry. By definition, if the position

Received 2019-03-27; accepted 2019-04-24; published 2019-07-01.

2010 Mathematics Subject Classification. 53A04, 53A07, 53A35.

Key words and phrases. Smarandache curves; hypersurfaces; Darboux frame; Euclidean 4-space.

(C)2019 Authors retain the copyrights of their papers, and all open access articles are distributed under the terms of the Creative Commons Attribution License. 
vector of a curve $\delta$ is composed by Frenet frame's vectors of another curve $\beta$, then the curve $\delta$ is called a Smarandache curve [2].

In differential geometry, frame fields constitute an important tool while studying curves and surfaces. The most familiar frame fields are Frenet frame along a space curve and Darboux frame along a surface curve [3]. It is analogous to the Frenet frame as applied to surface geometry. The Darboux frame exists at any nonumbilic point of a surface embedded in Euclidean space. In [4] M. Düldü et al. extend the Darboux frame field into Euclidean 4-space $\mathbb{E}^{4}$.

In the light of the existing studies in the field of geometry, many interesting results on Smarandache curves have been obtained by many mathematicians, see for example, [2, 3, 5-12]. Turgut and Yilmaz [11] have introduced a particular circumstance of such curves, they entitled it Smarandache $\mathbf{T B}_{2}$ curves in the space $\mathbb{E}_{4}^{1}$. They studied special Smarandache curves which are defined by the tangent and second binormal vector fields. In [8], the author has illustrated certain special Smarandache curves in the Euclidean 3-space.

Recently, H.S. Abdel-Aziz and M. Khalifa Saad [2,5] have studied special Smarandache curves of an arbitrary curve such as TN, TB and TNB with respect to Frenet frame in the three-dimensional Galilean and pseudo-Galilean spaces. Also, in $[6,7]$ they have investigated Smarandache curves according to Darboux frame in the three-dimensional Minkowski space $\mathbb{E}_{1}^{3}$.

The main goal of this article is to investigate Smarandache curves in $\mathbb{E}^{4}$ for a given curve with reference to its Darboux frame of first kind. The results presented in this paper generalize and refine some of the existing results in the literature and significant in mathematical modeling and other applications.

\section{BASIC NOTIONS AND PROPERTIES}

We now review some basic concepts on classical differential geometry of space curves and surfaces in Euclidean 4-space $\mathbb{E}^{4}[4,13-15]$. Let $\left\{e_{i} \mid i=1,2,3,4\right\}$ be the standard basis of $\mathbb{E}^{4}$, therefore $\mathbb{E}^{4}=\{X=$ $\left.\sum_{i=1}^{4} x_{i} e_{i} \mid x_{i} \in \mathbb{R}\right\}$. The scalar product and vector product of vectors $X, Y, Z \in \mathbb{E}^{4}$ are respectively defined by

$$
\begin{gathered}
\langle X, Y\rangle=\sum_{i=1}^{4} x_{i} y_{i}, \\
X \times Y \times Z=\left|\begin{array}{cccc}
e_{1} & e_{2} & e_{3} & e_{4} \\
x_{1} & x_{2} & x_{3} & x_{4} \\
y_{1} & y_{2} & y_{3} & y_{4} \\
z_{1} & z_{2} & z_{3} & z_{4}
\end{array}\right| .
\end{gathered}
$$

Definition 2.1. Let $f: D \subset \mathbb{E}^{4} \rightarrow \mathbb{R}$ be a differentiable mapping of an open set D. Given c $\in \mathbb{R}$, we recall that the level set $c$ of the $f$ is the set defined as $f^{-1}(c)$ which is the set of solutions in $D$ of the equations $f(x, y, z, w)=c$. 
Proposition 2.1. Consider that $f: U \subset \mathbb{E}^{4} \rightarrow \mathbb{R}$ is a differentiable function and $c \in f(U)$ is a regular value of $f$, then $f^{-1}(c)$ is a regular surface in $\mathbb{E}^{4}$. The implicit surface $f$ is regular if $\nabla f=\left(f_{x}, f_{y}, f_{z}, f_{w}\right) \neq 0$. The unit surface normal vector of the implicit surface $f$ is given by $\mathbf{N}=\frac{\nabla f}{\|\nabla f\|}$.

Definition 2.2. Suppose that $f: \mathbb{R}^{n} \rightarrow \mathbb{R}$, if all second partial derivatives of $f$ exist and are continuous on the domain of $f$, then the Hessian matrix $H_{f}$ is a square $n \times n$ matrix, usually defined as $H_{i j}=\frac{\partial^{2} f}{\partial x_{i} \partial x_{j}}$.

2.1. Curves on a hypersurface in $\mathbb{E}^{4}$.

Let $r: I \subset \mathbb{R} \rightarrow M$ be a regular curve in $\mathbb{E}^{4}$, where $\left\|r^{\prime}\right\|=\|d r / d u\|=1, \forall u \in I$, we will omit $u$ for simplification. Then the Frenet frame is defined by

$$
\left(\begin{array}{c}
\mathbf{t}^{\prime} \\
\mathbf{n}^{\prime} \\
\mathbf{b}_{1}^{\prime} \\
\mathbf{b}_{2}^{\prime}
\end{array}\right)=\left(\begin{array}{cccc}
0 & \kappa_{1} & 0 & 0 \\
-\kappa_{1} & 0 & \kappa_{2} & 0 \\
0 & -\kappa_{2} & 0 & \kappa_{3} \\
0 & 0 & \kappa_{3} & 0
\end{array}\right)\left(\begin{array}{c}
\mathbf{t} \\
\mathbf{n} \\
\mathbf{b}_{1} \\
\mathbf{b}_{2}
\end{array}\right),\left({ }^{\prime}=\frac{d}{d u}\right)
$$

where $\mathbf{t}, \mathbf{n}, \mathbf{b}_{\mathbf{1}}$ and $\mathbf{b}_{\mathbf{2}}$ are the tangent, principal normal, first binormal and the second binormal vector fields of $r$, respectively and the functions $\kappa_{i} \mid i=1,2,3$, are the curvature functions of $r$.

Theorem 2.1. [2] Let $r: I \rightarrow \mathbb{E}^{4}$ be a regular curve. Then

$$
\mathbf{t}=\frac{r^{\prime}}{\left\|r^{\prime}\right\|}, \quad \mathbf{b}_{\mathbf{2}}=\frac{r^{\prime} \times r^{\prime \prime} \times r^{(3)}}{\left\|r^{\prime} \times r^{\prime \prime} \times r^{(3)}\right\|}, \mathbf{b}_{\mathbf{1}}=\frac{b_{2} \times r^{\prime} \times r^{\prime \prime}}{\left\|b_{2} \times r^{\prime} \times r^{\prime \prime}\right\|}, \mathbf{n}=\frac{b_{1} \times b_{2} \times r^{\prime}}{\left\|b_{1} \times b_{2} \times r^{\prime}\right\|},
$$

and

$$
\kappa_{1}=\frac{\left\langle n, r^{\prime \prime}\right\rangle}{\left\|r^{\prime}\right\|^{2}}, \kappa_{2}=\frac{\left\langle b_{1}, r^{(3)}\right\rangle}{\left\|r^{\prime}\right\|^{3} \kappa_{1}}, \kappa_{3}=\frac{\left\langle b_{2}, r^{(4)}\right\rangle}{\left\|r^{\prime}\right\|^{4} \kappa_{1} \kappa_{2}} .
$$

Definition 2.3. Let $r$ be a regular curve in $\mathbb{E}^{4}$, which is parameterized by arc length and $n$-times continuously differentiable. Then $r$ is called a Frenet curve, if at every point the vectors $r^{\prime}, r^{\prime \prime}, \ldots, r^{(n-1)}$ are linearly independent.

\subsection{Darboux frame field of first kind in $\mathbb{E}^{4}$.}

Let $M$ be an oriented hypersurface in $\mathbb{E}^{4}$ and $r$ be a Frenet curve of class $\mathcal{C}^{n}(n \geq 4)$ with arc-length parameter $u$ lying on $M$. We denote the unit tangent and unit normal vector fields of the curve by $\mathbf{T}$ and $\mathbf{N}$, and $\mathbf{P}, \mathbf{U} \in T_{u} M$.

Definition 2.4. Let $r: I \rightarrow \mathbb{E}^{4}$ be a regular parameterized curve lying on $M$ in $\mathbb{E}^{4}$, then the frame field $\{\mathbf{T}, \mathbf{P}, \mathbf{U}, \mathbf{N}\}$ along $r$ is called extended Darboux frame field of first kind if $\left\{\mathbf{T}, \mathbf{N}, r^{\prime \prime}\right\}$ is linearly independent, therefore $\mathbf{T}=\frac{r^{\prime}}{\left\|r^{\prime}\right\|}, \mathbf{N}=\frac{\nabla f}{\|\nabla f\|}, \mathbf{P}=\frac{r^{\prime \prime}-\left(r^{\prime \prime} . \mathbf{N}\right) \mathbf{N}}{\left\|r^{\prime \prime}-\left(r^{\prime \prime} \cdot \mathbf{N}\right) \mathbf{N}\right\|}, \mathbf{U}=[\mathbf{T}, \mathbf{N}, \mathbf{E}]$ 
The derivatives of the Darboux frame field of first kind in $\mathbb{E}^{4}$ are given by [16]

$$
\left(\begin{array}{c}
\mathbf{T}^{\prime} \\
\mathbf{P}^{\prime} \\
\mathbf{U}^{\prime} \\
\mathbf{N}^{\prime}
\end{array}\right)=\left(\begin{array}{cccc}
0 & \kappa_{g}^{1} & 0 & \kappa_{n} \\
-\kappa_{g}^{1} & 0 & \kappa_{g}^{2} & \tau_{g}^{1} \\
0 & -\kappa_{g}^{2} & 0 & \tau_{g}^{2} \\
-\kappa_{n} & -\tau_{g}^{1} & -\tau_{g}^{2} & 0
\end{array}\right)\left(\begin{array}{c}
\mathbf{T} \\
\mathbf{P} \\
\mathbf{U} \\
\mathbf{N}
\end{array}\right)
$$

where $\kappa_{n}, \kappa_{g}^{1}, \kappa_{g}^{2}, \tau_{g}^{1}$ and $\tau_{g}^{2}$ are real valued functions denote the normal curvature, geodesic curvatures and the geodesic torsions, respectively. These functions are given by

$$
\begin{aligned}
\kappa_{n} & =\left\langle\mathbf{T}^{\prime}, \mathbf{N}\right\rangle=\frac{1}{\|\nabla f\|}\left\langle r^{\prime \prime}, \nabla f\right\rangle=\frac{-1}{\|\nabla f\|}\left(r^{\prime} H_{f}\left(r^{\prime}\right)^{t}\right), \\
\kappa_{g}^{1} & =\left\langle\mathbf{T}^{\prime}, \mathbf{P}\right\rangle=\left(r^{\prime \prime}\left(r^{\prime \prime}\right)^{t}-\frac{1}{\|\nabla f\|^{2}}\left(r^{\prime} H_{f}\left(r^{\prime}\right)^{t}\right)^{2}\right)^{\frac{1}{2}}, \\
\kappa_{g}^{2} & =\left\langle\mathbf{P}^{\prime}, \mathbf{U}\right\rangle=\frac{1}{\left(\kappa_{g}^{1}\right)^{2} \nabla f}\left(m_{1}+\frac{1}{\|\nabla f\|^{2}}\left(r^{\prime} H_{f}\left(r^{\prime}\right)^{t}\right) m_{2}\right), \\
\tau_{g}^{1} & =\left\langle\mathbf{P}^{\prime}, \mathbf{N}\right\rangle=\frac{-1}{\left(\kappa_{g}^{1}\right)}\left(\frac{1}{\|\nabla f\|}\left(r^{\prime} H_{f}\left(r^{\prime \prime}\right)^{t}\right)+\frac{1}{\|\nabla f\|^{3}}\left(r^{\prime} H_{f}(\nabla f)^{t}\right)\left(r^{\prime} H_{f}\left(r^{\prime}\right)^{t}\right)\right), \\
\tau_{g}^{2} & =\left\langle\mathbf{U}^{\prime}, \mathbf{N}\right\rangle=\left(\frac{-1}{\left(\kappa_{g}^{1}\right)\|\nabla f\|^{2}}\right) m_{3},
\end{aligned}
$$

where

$$
m_{1}=\left|\begin{array}{cccc}
x^{\prime} & y^{\prime} & z^{\prime} & w^{\prime} \\
x^{\prime \prime} & y^{\prime \prime} & z^{\prime \prime} & w^{\prime \prime} \\
x^{(3)} & y^{(3)} & z^{(3)} & w^{(3)} \\
f_{x} & f_{y} & f_{z} & f_{w}
\end{array}\right|, m_{2}=\left|\begin{array}{cccc}
x^{\prime} & y^{\prime} & z^{\prime} & w^{\prime} \\
x^{\prime \prime} & y^{\prime \prime} & z^{\prime \prime} & w^{\prime \prime} \\
a & b & c & d \\
f_{x} & f_{y} & f_{z} & f_{w}
\end{array}\right|, m_{3}=\left|\begin{array}{cccc}
x^{\prime} & y^{\prime} & z^{\prime} & w^{\prime} \\
f_{x} & f_{y} & f_{z} & f_{w} \\
a & b & c & d \\
p & q & t & v
\end{array}\right|,
$$

and

$$
\begin{aligned}
{[a, b, c, d] } & =(\nabla f)^{\prime}=r^{\prime} H_{f},[p, q, t, v]=(\nabla f)^{\prime \prime}=\alpha^{\prime \prime} H_{f}+\alpha^{\prime} \frac{d H_{f}^{\prime}}{d u} \\
\frac{d H_{f}}{d u} & =\left[\frac{\partial H_{f}}{\partial x}\left(r^{\prime}\right)^{t} \ldots \frac{\partial H_{f}}{\partial w}\left(r^{\prime}\right)^{t}\right] .
\end{aligned}
$$

Remark 2.1. [2] Let $r: I \rightarrow \mathbb{E}^{4}$ be a regular parameterized curve in $\mathbb{E}^{4}$, then

- $r$ is called asymptotic curve if and only if $k n=0$.

- $r$ is called a line of curvature if and only if $\tau_{g}^{1}=\tau_{g}^{2}=0$.

\section{First kind Smarandache CURVEs in $\mathbb{E}^{4}$}

Consider $r=r(u)$ is a curve lying fully on an oriented hypersurface $M$ in $\mathbb{E}^{4}$. Let $\{\mathbf{T}, \mathbf{P}, \mathbf{U}, \mathbf{N}\}$ be a Darboux frame field of first kind along $r(u)$ and $\kappa_{n}, \kappa_{g}^{1}, \kappa_{g}^{2}, \tau_{g}^{1}, \tau_{g}^{2}$ are real valued functions in arc length parameter $u$ of $r$. So, we have the following definition. 
Definition 3.1. [11] A regular curve $\alpha(s(u))$ in $\mathbb{E}^{4}$, whose position vector is obtained by extended Darboux frame vectors of another regular curve $r(u)$ is called Smarandache curve .

In the following we continue our studies of special Smarandache curves that we started in $[2,5,6]$. Here we investigate some special Smarandache curves of first kind called TP, TU, PU and PN ( the other special Smarandache curves can be computed in the same manner) and then obtain some of their differential geometric properties which represent the main results.

Let $r(u)=(x(u), y(u), z(u), w(u))$ be a curve of class $C^{n}(n \geq 4)$ lying on $M$. Then, by using proposition 2.1 , the unit normal vector field along $r$ is given by

$$
\overline{\mathbf{N}}=\frac{\nabla f}{\|\nabla f\|}
$$

\subsection{TP-Smarandache curves.}

Definition 3.2. Let $M$ be an oriented hypersurface in $\mathbb{E}^{4}$ and the Frent curve $r=r(u)$ lying fully on $M$ with Darboux frame $\{\mathbf{T}, \mathbf{P}, \mathbf{U}, \mathbf{N}\}$ and non-zero curvatures $\kappa_{n}, \kappa_{g}^{1}, \kappa_{g}^{2}, \tau_{g}^{1}$ and $\tau_{g}^{2}$. Then the $\mathbf{T P}$-Smarandache curve of $r$ is defined as

$$
\alpha(s)=\frac{1}{\sqrt{2}}(\mathbf{T}+\mathbf{P}) .
$$

Theorem 3.1. Let $r=r(u)$ be a Frenet curve lying on a hypersurface $M$ in $\mathbb{E}^{4}$ with Darboux frame $\{\mathbf{T}, \mathbf{P}, \mathbf{U}, \mathbf{N}\}$ and non-zero constant curvatures $\kappa_{n}, \kappa_{g}^{1}, \kappa_{g}^{2}, \tau_{g}^{1}$ and $\tau_{g}^{2}$. Then the curvature functions of the $\mathbf{T P}$ - Smarandache curve of $r$ satisfy the following equations:

$$
\begin{gathered}
\overline{\kappa_{n}}=\frac{1}{\lambda_{1}\|\nabla f\|}\left(\begin{array}{c}
\left(\kappa_{g}^{1}\left(-\kappa_{n}+\tau_{g}^{1}\right)+\kappa_{g}^{2} \tau_{g}^{2}\right) f_{w}-\left(\left(\kappa_{g}^{1}\right)^{2}+\right. \\
\left.\kappa_{n}\left(\kappa_{n}+\tau_{g}^{1}\right)\right) f_{x}-\left(\left(\kappa_{g}^{1}\right)^{2}+\left(\kappa_{g}^{2}\right)^{2}+\right. \\
\left.\tau_{g}^{1}\left(\kappa_{n}+\tau_{g}^{1}\right)\right) f_{y}+\left(\kappa_{g}^{1} \kappa_{g}^{2}-\left(\kappa_{n}+\tau_{g}^{1}\right) \tau_{g}^{2}\right) f_{z}
\end{array}\right), \\
\overline{\kappa_{g}^{1}}=\frac{1}{\lambda_{1} \lambda_{6}}\left(\begin{array}{c}
\left(\kappa_{g}^{1}\left(-\kappa_{n}+\tau_{g}^{1}\right)+\kappa_{g}^{2} \tau_{g}^{2}\right) \lambda_{5}+\left(\left(\kappa_{g}^{1}\right)^{2}+\right. \\
\left.\kappa_{n}\left(\kappa_{n}+\tau_{g}^{1}\right)\right) \lambda_{2}+\left(\left(\kappa_{g}^{1}\right)^{2}+\left(\kappa_{g}^{2}\right)^{2}+\right. \\
\left.\tau_{g}^{1}\left(\kappa_{n}+\tau_{g}^{1}\right)\right) \lambda_{3}+\left(\kappa_{g}^{1} \kappa_{g}^{2}-\left(\kappa_{n}+\tau_{g}^{1}\right) \tau_{g}^{2}\right) \lambda_{4}
\end{array}\right), \\
\overline{\kappa_{g}^{2}}=\frac{1}{\lambda_{6}^{2} \lambda_{11}}\left(\begin{array}{c} 
\\
-\left(\lambda_{2} \lambda_{7}+\lambda_{3} \lambda_{8}+\lambda_{4} \lambda_{9}+\lambda_{5} \lambda_{10}\right) \lambda_{6}^{\prime}+ \\
\lambda_{6}\left(\left(\kappa_{g}^{1} \lambda_{2}-\kappa_{g}^{2} \lambda_{4}\right) \lambda_{8}-\lambda_{5}\left(\kappa_{n} \lambda_{7}+\tau_{g}^{1} \lambda_{8}+\right.\right. \\
\left.\tau_{g}^{2} \lambda_{9}\right)+\lambda_{3}\left(-\kappa_{g}^{1} \lambda_{7}+\kappa_{g}^{2} \lambda_{9}+\tau_{g}^{1} \lambda_{10}\right)+\lambda_{7} \lambda_{2}^{\prime}+ \\
\left.\lambda_{8} \lambda_{3}^{\prime}+\lambda_{9} \lambda_{4}^{\prime}+\lambda_{10}\left(\kappa_{n} \lambda_{2}+\tau_{g}^{2} \lambda_{4}+\lambda_{5}^{\prime}\right)\right)
\end{array}\right),
\end{gathered}
$$




$$
\begin{gathered}
\overline{\tau_{g}^{1}}=\frac{1}{\lambda_{6}^{2}\|\nabla f\|}\left(\begin{array}{c}
-\left(f_{x} \lambda_{2}+f_{y} \lambda_{3}+f_{z} \lambda_{4}+f_{w} \lambda_{5}\right) \lambda_{6}^{\prime}+ \\
\lambda_{6}\left(-\kappa_{g}^{1} f_{x} \lambda_{3}+\kappa_{g}^{2} f_{z} \lambda_{3}-\kappa_{n} f_{x} \lambda_{5}-\right. \\
\tau_{g}^{2} f_{z} \lambda_{5}+f_{x} \lambda_{2}^{\prime}+f_{y}\left(\kappa_{g}^{1} \lambda_{2}-\kappa_{g}^{2} \lambda_{4}-\tau_{g}^{1} \lambda_{5}+\right. \\
\left.\left.\lambda_{3}^{\prime}\right)+f_{z} \lambda_{4}^{\prime}+f_{w}\left(\kappa_{n} \lambda_{2}+\tau_{g}^{1} \lambda_{3}+\tau_{g}^{2} \lambda_{4}+\lambda_{5}^{\prime}\right)\right)
\end{array}\right), \\
\overline{\tau_{g}^{2}=} \frac{1}{\lambda_{11}^{2}\|\nabla f\|}\left(\begin{array}{c}
-\left(f_{x} \lambda_{7}+f_{y} \lambda_{8}+f_{z} \lambda_{9}+f_{w} \lambda_{10}\right) \lambda_{11}^{\prime}+ \\
\lambda_{11}\left(-\kappa_{g}^{1} f_{x} \lambda_{8}+\kappa_{g}^{2} f_{z} \lambda_{8}-\kappa_{n} f_{x} \lambda_{10}-\right. \\
\tau_{g}^{2} f_{z} \lambda_{10}+f_{x} \lambda_{7}^{\prime}+f_{y}\left(\kappa_{g}^{1} \lambda_{7}-\kappa_{g}^{2} \lambda_{9}-\tau_{g}^{1} \lambda_{10}+\right. \\
\left.\left.\lambda_{8}^{\prime}\right)+f_{z} \lambda_{9}^{\prime}+f_{w}\left(\kappa_{n} \lambda_{7}+\tau_{g}^{1} \lambda_{8}+\tau_{g}^{2} \lambda_{9}+\lambda_{10}^{\prime}\right)\right)
\end{array}\right) .
\end{gathered}
$$

Proof. Because $\alpha=\alpha(s)$ is a $\mathbf{T P}-$ Smarandache curve reference to Frenet curve $r$, then differentiating Eq. (3.2), we get

$$
\alpha^{\prime}=\frac{1}{\sqrt{2}}\left(-\kappa_{g}^{1} \mathbf{T}+\kappa_{g}^{1} \mathbf{P}+\kappa_{g}^{2} \mathbf{U}+\left(\tau_{g}^{1}+\kappa_{n}\right) \mathbf{N}\right) .
$$

Again, differentiating Eq. (3.4), we obtain

$$
\alpha^{\prime \prime}=\frac{1}{\sqrt{2}}\left(\begin{array}{c}
\left(-\left(\kappa_{g}^{1}\right)^{2}-\kappa_{n}\left(\kappa_{n}+\tau_{g}^{1}\right)\right) \mathbf{T}-\left(\left(\kappa_{g}^{1}\right)^{2}+\left(\kappa_{g}^{2}\right)^{2}+\tau_{g}^{1}\left(\kappa_{n}+\tau_{g}^{1}\right)\right) \mathbf{P} \\
+\left(\kappa_{g}^{1} \kappa_{g}^{2}-\left(\kappa_{n}+\tau_{g}^{1}\right) \tau_{g}^{2}\right) \mathbf{U}+\left(-\kappa_{g}^{1} \kappa_{n}+\kappa_{g}^{1} \tau_{g}^{1}+\kappa_{g}^{2} \tau_{g}^{2}\right) \mathbf{N}
\end{array}\right) .
$$

Also, Eq. (3.4) leads to

$$
\overline{\mathbf{T}}=\frac{-\kappa_{g}^{1} \mathbf{T}+\kappa_{g}^{1} \mathbf{P}+\kappa_{g}^{2} \mathbf{U}+\left(\tau_{g}^{1}+\kappa_{n}\right) \mathbf{N}}{\lambda_{1}},
$$

where

$$
\lambda_{1}=\sqrt{2\left(\kappa_{g}^{1}\right)^{2}+\left(\kappa_{g}^{2}\right)^{2}+\left(\tau_{g}^{1}+\kappa_{n}\right)^{2}},
$$

and we get

$$
\overline{\mathbf{N}}=\frac{f_{x} \mathbf{T}+f_{y} \mathbf{P}+f_{z} \mathbf{U}+f_{w} \mathbf{N}}{\|\nabla f\|} .
$$

On the other hand, we have

$$
\overline{\mathbf{P}}=\frac{\lambda_{2} \mathbf{T}+\lambda_{3} \mathbf{P}+\lambda_{4} \mathbf{U}+\lambda_{5} \mathbf{N}}{\lambda_{6}},
$$

where

$$
\begin{aligned}
\lambda_{2}= & \frac{1}{\sqrt{2}}\left(\left(\kappa_{g}^{1}\right)^{2}+\left(\kappa_{n}\right)^{2}+\kappa_{n} \tau_{g}^{1}\right)+\frac{\sigma_{1}}{\|\nabla f\|} f_{x}, \\
\lambda_{3}= & \frac{1}{\sqrt{2}}\left(\left(\kappa_{g}^{1}\right)^{2}+\left(\kappa_{g}^{2}\right)^{2}+\kappa_{n} \tau_{g}^{1}+\left(\tau_{g}^{1}\right)^{2}\right)+\frac{\sigma_{1}}{\|\nabla f\|} f_{y}, \\
\lambda_{4}= & \frac{1}{\sqrt{2}}\left(-\kappa_{g}^{1} \kappa_{g}^{2}+\kappa_{n} \tau_{g}^{2}+\tau_{g}^{1} \tau_{g}^{2}\right)+\frac{\sigma_{1}}{\|\nabla f\|} f_{z}, \\
\lambda_{5}= & \frac{1}{\sqrt{2}}\left(\kappa_{g}^{1} \kappa_{n}-\kappa_{g}^{1} \tau_{g}^{1}-\kappa_{g}^{2} \tau_{g}^{2}\right)+\frac{\sigma_{1}}{\|\nabla f\|} f_{w}, \\
& \lambda_{6}=\sqrt{\left(\lambda_{2}\right)^{2}+\left(\lambda_{3}\right)^{2}+\left(\lambda_{4}\right)^{2}+\left(\lambda_{5}\right)^{2}},
\end{aligned}
$$




$$
\sigma_{1}=\left\langle\alpha^{\prime \prime}, \mathbf{N}\right\rangle=\frac{1}{\sqrt{2}\|\nabla f\|}\left(\begin{array}{c}
\left(-\left(\kappa_{g}^{1}\right)^{2}-\kappa_{n}\left(\kappa_{n}+\tau_{g}^{1}\right)\right) f_{x}-\left(\left(\kappa_{g}^{1}\right)^{2}+\left(\kappa_{g}^{2}\right)^{2}+\right. \\
\left.\tau_{g}^{1}\left(\kappa_{n}+\tau_{g}^{1}\right)\right) f_{y}+\left(\kappa_{g}^{1} \kappa_{g}^{2}-\left(\kappa_{n}+\tau_{g}^{1}\right) \tau_{g}^{2}\right) f_{z}+ \\
\left(-\kappa_{g}^{1} \kappa_{n}+\kappa_{g}^{1} \tau_{g}^{1}+\kappa_{g}^{2} \tau_{g}^{2}\right) f_{w}
\end{array}\right)
$$

Also, we get

$$
\overline{\mathbf{U}}=\frac{\lambda_{7} \mathbf{T}+\lambda_{8} \mathbf{P}+\lambda_{9} \mathbf{U}+\lambda_{10} \mathbf{N}}{\lambda_{11}}
$$

where,

$$
\begin{aligned}
& \lambda_{7}=\left(\begin{array}{c}
\kappa_{g}^{2} f_{w} \lambda_{3}-\kappa_{n} f_{z} \lambda_{3}-\tau_{g}^{1} f_{z} \lambda_{3}-\kappa_{g}^{1} f_{w} \lambda_{4}+ \\
\kappa_{n} f_{y} \lambda_{4}+\tau_{g}^{1} f_{y} \lambda_{4}-\kappa_{g}^{2} f_{y} \lambda_{5}+\kappa_{g}^{1} f_{z} \lambda_{5}
\end{array}\right), \\
& \lambda_{8}=\left(\begin{array}{c}
-\kappa_{g}^{2} f_{w} \lambda_{2}+\kappa_{n} f_{z} \lambda_{2}+\tau_{g}^{1} f_{z} \lambda_{2}-\kappa_{g}^{1} f_{w} \lambda_{4}- \\
\kappa_{n} f_{x} \lambda_{4}-\tau_{g}^{1} f_{x} \lambda_{4}+\kappa_{g}^{2} f_{x} \lambda_{5}+\kappa_{g}^{1} f_{z} \lambda_{5}
\end{array}\right), \\
& \lambda_{9}=\left(\begin{array}{c}
\kappa_{g}^{1} f_{w} \lambda_{2}-\kappa_{n} f_{y} \lambda_{2}-\tau_{g}^{1} f_{y} \lambda_{2}+\kappa_{g}^{1} f_{w} \lambda_{3}+ \\
\kappa_{n} f_{x} \lambda_{3}+\tau_{g}^{1} f_{x} \lambda_{3}-\kappa_{g}^{1} F_{x} \lambda_{5}-\kappa_{g}^{1} F_{y} \lambda_{5}
\end{array}\right), \\
& \lambda_{10}=\left(\begin{array}{c}
\kappa_{g}^{2} f_{y} \lambda_{2}-\kappa_{g}^{1} f_{z} \lambda_{2}-\kappa_{g}^{2} f_{x} \lambda_{3}-\kappa_{g}^{1} f_{z} \lambda_{3}+\kappa_{g}^{1} f_{x} \lambda_{4}+\kappa_{g}^{1} f_{y} \lambda_{4}
\end{array}\right), \\
& \lambda_{11}=\lambda_{1} \lambda_{6}\|\nabla f\| .
\end{aligned}
$$

In the light of the above calculations, the curvature functions $\overline{\kappa_{n}}, \overline{\kappa_{g}^{1}}, \overline{\kappa_{g}^{2}}, \overline{\tau_{g}^{1}}$ and $\overline{\tau_{g}^{2}}$ of $\alpha$ are computed as in Eqs. (3.3).

Corollary 3.1. If $r$ is an asymptotic curve. Then, the following equations hold:

$$
\begin{aligned}
& \overline{\mathbf{T}}=\frac{-\kappa_{g}^{1} \mathbf{T}+\kappa_{g}^{1} \mathbf{P}+\kappa_{g}^{2} \mathbf{U}+\tau_{g}^{1} \mathbf{N}}{\lambda_{1}}, \overline{\mathbf{N}}=\frac{f_{x} \mathbf{T}+f_{y} \mathbf{P}+f_{z} \mathbf{U}+f_{w} \mathbf{N}}{\|\nabla f\|}, \\
& \overline{\mathbf{P}}=\frac{\lambda_{13} \mathbf{T}+\lambda_{14} \mathbf{P}+\lambda_{15} \mathbf{U}+\lambda_{16} \mathbf{N}}{\lambda_{17}}, \overline{\mathbf{U}}=\frac{\lambda_{18} \mathbf{T}+\lambda_{19} \mathbf{P}+\lambda_{20} \mathbf{U}+\lambda_{21} \mathbf{N}}{\lambda_{22}},
\end{aligned}
$$

and then the curvature functions are computed as follows

$$
\begin{aligned}
\overline{\kappa_{n}=} & \frac{\left(\kappa_{g}^{1} \tau_{g}^{1}+\kappa_{g}^{2} \tau_{g}^{2}\right) f_{w}-\left(\kappa_{g}^{1}\right)^{2} f_{x}-\left(\left(\kappa_{g}^{1}\right)^{2}+\left(\kappa_{g}^{2}\right)^{2}+\left(\tau_{g}^{1}\right)^{2}\right) f_{y}+\left(\kappa_{g}^{1} \kappa_{g}^{2}-\tau_{g}^{1} \tau_{g}^{2}\right) f_{z}}{\lambda_{12}\|\nabla f\|} \\
\overline{\kappa_{g}^{1}=} & \frac{\left(\kappa_{g}^{1} \tau_{g}^{1}+\kappa_{g}^{2} \tau_{g}^{2}\right) \lambda_{16}-\left(\kappa_{g}^{1}\right)^{2} \lambda_{13}-\left(\left(\kappa_{g}^{1}\right)^{2}+\left(\kappa_{g}^{2}\right)^{2}+\left(\tau_{g}^{1}\right)^{2}\right) \lambda_{14}+\left(\kappa_{g}^{1} \kappa_{g}^{2}-\tau_{g}^{1} \tau_{g}^{2}\right) \lambda_{15}}{\lambda_{12} \lambda_{17}} \\
& \overline{\kappa_{g}^{2}}=\frac{1}{\lambda_{22} \lambda_{17}^{2}}\left(\begin{array}{c}
\lambda_{17}\left(\kappa_{g}^{1} \lambda_{13} \lambda_{19}-\kappa_{g}^{2} \lambda_{15} \lambda_{19}-\tau_{g}^{1} \lambda_{16} \lambda_{19}-\right. \\
\tau_{g}^{2} \lambda_{16} \lambda_{20}+\lambda_{14}\left(-\kappa_{g}^{1} \lambda_{18}+\kappa_{g}^{2} \lambda_{20}\right)+\lambda_{18} \lambda_{13}^{\prime}+ \\
\lambda_{19} \lambda_{14}^{\prime}+\lambda_{20} \lambda_{15}^{\prime}+\lambda_{21}\left(\tau_{g}^{1} \lambda_{14}+\tau_{g}^{2} \lambda_{15}+\right. \\
\left.\left.\lambda_{16}^{\prime}\right)\right)-\left(\lambda_{21} \lambda_{16}+\lambda_{13} \lambda_{18}+\lambda_{14} \lambda_{19}+\lambda_{15} \lambda_{20}\right) \lambda_{17}^{\prime}
\end{array}\right)
\end{aligned}
$$




$$
\begin{gathered}
\overline{\tau_{g}^{1}}=\frac{1}{\lambda_{17}^{2}\|\nabla f\|}\left(\begin{array}{c}
-f_{x}\left(\kappa_{g}^{1} \lambda_{14} \lambda_{17}-\lambda_{17} \lambda_{13}^{\prime}+\lambda_{13} \lambda_{17}^{\prime}\right)+f_{y}\left(\lambda _ { 1 7 } \left(\kappa_{g}^{1} \lambda_{13}-\right.\right. \\
\left.\left.\kappa_{g}^{2} \lambda_{15}-\tau_{g}^{1} \lambda_{16}+\lambda_{14}^{\prime}\right)-\lambda_{14} \lambda_{17}^{\prime}\right)+ \\
f_{z}\left(\lambda_{17}\left(\kappa_{g}^{2} \lambda_{14}-\tau_{g}^{2} \lambda_{16}+\lambda_{15}^{\prime}\right)-\lambda_{15} \lambda_{17}^{\prime}\right)+ \\
f_{w}\left(\lambda_{17}\left(\tau_{g}^{1} \lambda_{14}+\tau_{g}^{2} \lambda_{15}+\lambda_{16}^{\prime}\right)-\lambda_{16} \lambda_{17}^{\prime}\right)
\end{array}\right), \\
\overline{\tau_{g}^{2}}=\frac{1}{\lambda_{22}^{2}\|\nabla f\|}\left(\begin{array}{c}
-\left(\lambda_{21} f_{w}+\lambda_{18} f_{x}+\lambda_{19} f_{y}+\lambda_{20} f_{z}\right) \lambda_{22}^{\prime}+\lambda_{22}\left(\left(-\tau_{g}^{1} \lambda_{21}+\right.\right. \\
\left.\kappa_{g}^{1} \lambda_{18}\right) f_{y}+\lambda_{20}\left(\tau_{g}^{2} f_{w}-\kappa_{g}^{2} f_{y}\right)+\lambda_{19}\left(\tau_{g}^{1} f_{w}-\kappa_{g}^{1} f_{x}+\right. \\
\left.\left.\kappa_{g}^{2} f_{z}\right)+f_{w} \lambda_{21}^{\prime}+f_{x} \lambda_{18}^{\prime}+f_{y} \lambda_{19}^{\prime}+f_{z}\left(-\tau_{g}^{2} \lambda_{21}+\lambda_{20}^{\prime}\right)\right)
\end{array}\right),
\end{gathered}
$$

where

$$
\begin{aligned}
& \lambda_{12}=\sqrt{2\left(\kappa_{g}^{1}\right)^{2}+\left(\kappa_{g}^{2}\right)^{2}+\left(\tau_{g}^{1}\right)^{2}}, \\
& \lambda_{13}=\sqrt{2}\left(\kappa_{g}^{1}\right)^{2}\|\nabla f\|+2 \sigma_{1} f_{x}, \\
& \lambda_{14}=\sqrt{2}\left(\left(\kappa_{g}^{1}\right)^{2}+\left(\kappa_{g}^{2}\right)^{2}+\left(\tau_{g}^{1}\right)^{2}\right)\|\nabla f\|+2 \sigma_{1} f_{y}, \\
& \lambda_{15}=\sqrt{2}\left(-\kappa_{g}^{1} \kappa_{g}^{2}+\tau_{g}^{1} \tau_{g}^{2}\right)\|\nabla f\|+2 \sigma_{1} f_{z}, \\
& \lambda_{16}=\sqrt{2}\left(-\kappa_{g}^{1} \tau_{g}^{1}-\kappa_{g}^{2} \tau_{g}^{2}\right)\|\nabla f\|+2 \sigma_{1} f_{w}, \\
& \lambda_{17}=\sqrt{\left(\lambda_{13}\right)^{2}+\left(\lambda_{14}\right)^{2}+\left(\lambda_{15}\right)^{2}+\left(\lambda_{16}\right)^{2}}, \\
& \lambda_{18}=\kappa_{g}^{2} f_{w} \lambda_{14}-\tau_{g}^{1} f_{z} \lambda_{14}-\kappa_{g}^{1} f_{w} \lambda_{15}+\tau_{g}^{1} f_{y} \lambda_{15}-\kappa_{g}^{2} f_{y} \lambda_{16}+\kappa_{g}^{1} f_{z} \lambda_{16}, \\
& \lambda_{19}=-\kappa_{g}^{2} f_{w} \lambda_{13}+\tau_{g}^{1} f_{z} \lambda_{13}-\kappa_{g}^{1} f_{w} \lambda_{15}-\tau_{g}^{1} f_{x} \lambda_{15}+\kappa_{g}^{2} f_{x} \lambda_{16}+\kappa_{g}^{1} f_{z} \lambda_{16}, \\
& \lambda_{20}=k_{g}^{1} \lambda_{13} f_{w}+k_{g}^{1} \lambda_{14} f_{w}+\tau_{g}^{1} \lambda_{14} f_{x}-k_{g}^{1} \lambda_{16} f_{x}-\tau_{g}^{1} \lambda_{13} f_{y}-k_{g}^{1} \lambda_{16} f_{y}, \\
& \lambda_{21}=\kappa_{g}^{2} f_{y} \lambda_{13}-\kappa_{g}^{1} f_{z} \lambda_{13}-\kappa_{g}^{2} f_{x} \lambda_{14}-\kappa_{g}^{1} f_{z} \lambda_{14}+\kappa_{g}^{1} f_{x} \lambda_{15}+\kappa_{g}^{1} f_{y} \lambda_{15}, \\
& \lambda_{22}=\lambda_{12} \lambda_{17}\|\nabla f\|, \\
& \sigma_{1}=\frac{1}{\sqrt{2}\|\nabla f\|}\left(\begin{array}{c}
-\left(\kappa_{g}^{1}\right)^{2} f_{x}-\left(\left(\kappa_{g}^{1}\right)^{2}+\left(\kappa_{g}^{2}\right)^{2}+\left(\tau_{g}^{1}\right)^{2}\right) f_{y}+ \\
\left(\kappa_{g}^{1} \kappa_{g}^{2}-\tau_{g}^{1} \tau_{g}^{2}\right) f_{z}+\left(\kappa_{g}^{1} \tau_{g}^{1}+\kappa_{g}^{2} \tau_{g}^{2}\right) f_{w}
\end{array}\right) .
\end{aligned}
$$

Corollary 3.2. If $r$ is a line of curvature. Then, the following equations hold:

$$
\begin{aligned}
& \overline{\mathbf{T}}=\frac{-\kappa_{g}^{1} \mathbf{T}+\kappa_{g}^{1} \mathbf{P}+\kappa_{g}^{2} \mathbf{U}+\kappa_{n} \mathbf{N}}{\lambda_{1}}, \overline{\mathbf{N}}=\frac{f_{x} \mathbf{T}+f_{y} \mathbf{P}+f_{z} \mathbf{U}+f_{w} \mathbf{N}}{\|\nabla f\|}, \\
& \overline{\mathbf{P}}=\frac{\lambda_{24} \mathbf{T}+\lambda_{25} \mathbf{P}+\lambda_{26} \mathbf{U}+\lambda_{27} \mathbf{N}}{\lambda_{28}}, \overline{\mathbf{U}}=\frac{\lambda_{29} \mathbf{T}+\lambda_{30} \mathbf{P}+\lambda_{31} \mathbf{U}+\lambda_{32} \mathbf{N}}{\lambda_{33}},
\end{aligned}
$$

and then the curvature functions are computed as follows

$$
\begin{aligned}
\overline{\kappa_{n}} & =-\frac{\kappa_{g}^{1} \kappa_{n} f_{w}+\left(\left(\kappa_{g}^{1}\right)^{2}+\kappa_{n}^{2}\right) f_{x}+\left(\left(\kappa_{g}^{1}\right)^{2}+\left(\kappa_{g}^{2}\right)^{2}\right) f_{y}-\kappa_{g}^{1} \kappa_{g}^{2} f_{z}}{\lambda_{23}\|\nabla f\|}, \\
\overline{\kappa_{g}^{1}} & =-\frac{\kappa_{g}^{1} \kappa_{n} \lambda_{27}+\left(\left(\kappa_{g}^{1}\right)^{2}+\kappa_{n}^{2}\right) \lambda_{24}+\left(\left(\kappa_{g}^{1}\right)^{2}+\left(\kappa_{g}^{2}\right)^{2}\right) \lambda_{25}-\kappa_{g}^{1} \kappa_{g}^{2} \lambda_{26}}{\lambda_{23} \lambda_{28}},
\end{aligned}
$$




$$
\begin{gathered}
\overline{\kappa_{g}^{2}}=\frac{1}{\lambda_{33} \lambda_{28}^{2}}\left(\begin{array}{c}
\lambda_{28}\left(\lambda_{25}\left(-\kappa_{g}^{1} \lambda_{29}+\kappa_{g}^{2} \lambda_{31}\right)+\lambda_{29}\left(-\kappa_{n} \lambda_{27}+\lambda_{24}^{\prime}\right)+\right. \\
\lambda_{30}\left(\kappa_{g}^{1} \lambda_{24}-\kappa_{g}^{2} \lambda_{26}+\lambda_{25}^{\prime}\right)+\lambda_{31} \lambda_{26}^{\prime}+\lambda_{32}\left(\kappa_{n} \lambda_{24}+\right. \\
\left.\left.\lambda_{27}^{\prime}\right)\right)-\left(\lambda_{32} \lambda_{27}+\lambda_{24} \lambda_{29}+\lambda_{25} \lambda_{30}+\lambda_{26} \lambda_{31}\right) \lambda_{28}^{\prime}
\end{array}\right), \\
\overline{\tau_{g}^{1}}=\frac{1}{\lambda_{28}^{2}\|\nabla f\|}\left(\begin{array}{c}
-f_{x}\left(\lambda_{28}\left(\kappa_{g}^{1} \lambda_{25}+\kappa_{n} \lambda_{27}-\lambda_{24}^{\prime}\right)+\lambda_{24} \lambda_{28}^{\prime}\right)+ \\
f_{y}\left(\lambda_{28}\left(\kappa_{g}^{1} \lambda_{24}-\kappa_{g}^{2} \lambda_{26}+\lambda_{25}^{\prime}\right)-\lambda_{25} \lambda_{28}^{\prime}\right)+ \\
f_{z}\left(\lambda_{28}\left(\kappa_{g}^{2} \lambda_{25}+\lambda_{26}^{\prime}\right)-\lambda_{26} \lambda_{28}^{\prime}\right)+ \\
f_{w}\left(\lambda_{28}\left(\kappa_{n} \lambda_{24}+\lambda_{27}^{\prime}\right)-\lambda_{27} \lambda_{28}^{\prime}\right)
\end{array}\right), \\
\overline{\tau_{g}^{2}=} \frac{1}{\lambda_{33}^{2}\|\nabla f\|}\left(\begin{array}{c}
-\left(\lambda_{32} f_{w}+\lambda_{29} f_{x}+\lambda_{30} f_{y}+\lambda_{31} f_{z}\right) \lambda_{33}^{\prime}+ \\
\lambda_{33}\left(-\kappa_{n} \lambda_{32} f_{x}-\kappa_{g}^{1} \lambda_{30} f_{x}+\right. \\
\lambda_{29}\left(\kappa_{n} f_{w}+\kappa_{g}^{1} f_{y}\right)+\kappa_{g}^{2} \lambda_{30} f_{z}+f_{w} \lambda_{32}^{\prime}+ \\
\left.f_{x} \lambda_{29}^{\prime}+f_{y}\left(-\kappa_{g}^{2} \lambda_{31}+\lambda_{30}^{\prime}\right)+f_{z} \lambda_{31}^{\prime}\right)
\end{array}\right)
\end{gathered}
$$

where

$$
\begin{aligned}
& \lambda_{23}=\sqrt{2\left(\kappa_{g}^{1}\right)^{2}+\left(\kappa_{g}^{2}\right)^{2}+\left(\kappa_{n}\right)^{2}}, \lambda_{24}=\sqrt{2}\left(\left(\kappa_{g}^{1}\right)^{2}+\kappa_{n}^{2}\right)^{2}\|\nabla f\|+2 \sigma_{1} f_{x}, \\
& \lambda_{25}=\sqrt{2}\left(\left(\kappa_{g}^{1}\right)^{2}+\left(\kappa_{g}^{2}\right)^{2}\right)\|\nabla f\|+2 \sigma_{1} f_{y}, \lambda_{26}=-\sqrt{2} \kappa_{g}^{1} \kappa_{g}^{2}\|\nabla f\|+2 \sigma_{1} f_{z}, \\
& \lambda_{27}=\sqrt{2} \kappa_{g}^{1} \kappa_{n}\|\nabla f\|+2 \sigma_{1} f_{w}, \lambda_{28}=\sqrt{\left(\lambda_{24}\right)^{2}+\left(\lambda_{25}\right)^{2}+\left(\lambda_{26}\right)^{2}+\left(\lambda_{27}\right)^{2}} \\
& \lambda_{29}=\kappa_{g}^{2} f_{w} \lambda_{25}-\kappa_{n} f_{z} \lambda_{25}-\kappa_{g}^{1} f_{w} \lambda_{26}+\kappa_{n} f_{y} \lambda_{26}-\kappa_{g}^{2} f_{y} \lambda_{27}+\kappa_{g}^{1} f_{z} \lambda_{27}, \\
& \lambda_{30}=-\kappa_{g}^{2} f_{w} \lambda_{24}+\kappa_{n} f_{z} \lambda_{24}-\kappa_{g}^{1} f_{w} \lambda_{26}-\kappa_{n} f_{x} \lambda_{26}+\kappa_{g}^{2} f_{x} \lambda_{27}+\kappa_{g}^{1} f_{z} \lambda_{27}, \\
& \lambda_{31}=\kappa_{g}^{1} f_{w} \lambda_{24}-\kappa_{n} f_{y} \lambda_{24}+\kappa_{g}^{1} f_{w} \lambda_{25}+\kappa_{n} f_{x} \lambda_{25}-\kappa_{g}^{1} F_{x} \lambda_{27}-\kappa_{g}^{1} F_{y} \lambda_{27}, \\
& \lambda_{32}=\kappa_{g}^{2} f_{y} \lambda_{24}-\kappa_{g}^{1} f_{z} \lambda_{24}-\kappa_{g}^{2} f_{x} \lambda_{25}-\kappa_{g}^{1} f_{z} \lambda_{25}+\kappa_{g}^{1} f_{x} \lambda_{26}+\kappa_{g}^{1} f_{y} \lambda_{26}, \\
& \lambda_{33}=\lambda_{23} \lambda_{28}\|\nabla f\|, \\
& \sigma_{1}=\frac{1}{\sqrt{2}\|\nabla f\|}\left(-\kappa_{g}^{1} \kappa_{n} f_{w}-\left(\left(\kappa_{g}^{1}\right)^{2}+\kappa_{n}^{2}\right) f_{x}-\left(\left(\kappa_{g}^{1}\right)^{2}+\left(\kappa_{g}^{2}\right)^{2}\right) f_{y}+\kappa_{g}^{1} \kappa_{g}^{2} f_{z}\right) .
\end{aligned}
$$

\subsection{TU-Smarandache curves.}

Definition 3.3. Let $M$ be an oriented hypersurface in $\mathbb{E}^{4}$ and the Frenet curve $r=r(u)$ lying fully on $M$ with Darboux frame $\{\mathbf{T}, \mathbf{P}, \mathbf{U}, \mathbf{N}\}$ and non-zero curvatures $\kappa_{n}, \kappa_{g}^{1}, \kappa_{g}^{2}, \tau_{g}^{1}$ and $\tau_{g}^{2}$. Then the $\mathbf{T} \boldsymbol{U}$-Smarandache curve of $r$ is defined as

$$
\beta(u)=\frac{1}{\sqrt{2}}(\mathbf{T}+\mathbf{U})
$$

Theorem 3.2. Let $r=r(u)$ be a Frenet curve lying on a hypersurface $M$ in $\mathbb{E}^{4}$ with Darboux frame $\{\mathbf{T}, \mathbf{P}, \mathbf{U}, \mathbf{N}\}$ and non-zero constant curvatures; $\kappa_{n}, \kappa_{g}^{1}, \kappa_{g}^{2}, \tau_{g}^{1}$ and $\tau_{g}^{2}$. Then the curvature functions of the 
TU-Smarandache curve of $r$ satisfy the following equations:

$$
\begin{aligned}
& \overline{\kappa_{n}}=\frac{1}{\mu_{1}\|\nabla f\|}\left(\begin{array}{c}
\left(\kappa_{g}^{1}-\kappa_{g}^{2}\right) \tau_{g}^{1} f_{w}-\left(\kappa_{g}^{1}\left(\kappa_{g}^{1}-\kappa_{g}^{2}\right)+\kappa_{n}\left(\kappa_{n}+\tau_{g}^{2}\right)\right) f_{x}+ \\
\left(\kappa_{g}^{1}-\kappa_{g}^{2}\right) \kappa_{g}^{2} f_{y}-\left(\kappa_{n}+\tau_{g}^{2}\right)\left(\tau_{g}^{1} f_{y}+\tau_{g}^{2} f_{z}\right)
\end{array}\right), \\
& \bar{\kappa}_{g}^{1}=\frac{1}{\mu_{1} \mu_{6}}\left(\begin{array}{c}
\left(\kappa_{g}^{1}-\kappa_{g}^{2}\right) \tau_{g}^{1} \mu_{5}-\left(\kappa_{g}^{1}\left(\kappa_{g}^{1}-\kappa_{g}^{2}\right)+\kappa_{n}\left(\kappa_{n}+\tau_{g}^{2}\right)\right) \mu_{2}+ \\
\left(\kappa_{g}^{1}-\kappa_{g}^{2}\right) \kappa_{g}^{2} \mu_{3}-\left(\kappa_{n}+\tau_{g}^{2}\right)\left(\tau_{g}^{1} f_{y}+\tau_{g}^{2} \mu_{4}\right)
\end{array}\right), \\
& \overline{\kappa_{g}^{2}}=\frac{1}{\mu_{6}^{2} \mu_{11}}\left(\begin{array}{c}
-\left(\mu_{2} \mu_{7}+\mu_{3} \mu_{8}+\mu_{4} \mu_{9}+\mu_{5} \mu_{10}\right) \mu_{6}^{\prime}+\mu_{6}\left(\left(\kappa_{g}^{1} \mu_{2}-\right.\right. \\
\left.\kappa_{g}^{2} \mu_{4}\right) \mu_{8}-\mu_{5}\left(\kappa_{n} \mu_{7}+\tau_{g}^{1} \mu_{8}+\tau_{g}^{2} \mu_{9}\right)+\mu_{3}\left(-\kappa_{g}^{1} \mu_{7}+\right. \\
\left.\kappa_{g}^{2} \mu_{9}+\tau_{g}^{1} \mu_{10}\right)+\mu_{7} \mu_{2}^{\prime}+\mu_{8} \mu_{3}^{\prime}+ \\
\left.\mu_{9} \mu_{4}^{\prime}+\mu_{10}\left(\kappa_{n} \mu_{2}+\tau_{g}^{2} \mu_{4}+\mu_{5}^{\prime}\right)\right)
\end{array}\right) \text {, } \\
& \overline{\tau_{g}^{1}}=\frac{1}{\mu_{6}^{2}\|\nabla f\|}\left(\begin{array}{c}
-\left(f_{x} \mu_{2}+f_{y} \mu_{3}+f_{z} \mu_{4}+f_{w} \mu_{5}\right) \mu_{6}^{\prime}+\mu_{6}\left(f_{x} \mu_{2}^{\prime}\right. \\
-\kappa_{g}^{1} f_{x} \mu_{3}+\kappa_{g}^{2} f_{z} \mu_{3}-\kappa_{n} f_{x} \mu_{5}-\tau_{g}^{2} f_{z} \mu_{5}+ \\
f_{y}\left(\kappa_{g}^{1} \mu_{2}-\kappa_{g}^{2} \mu_{4}-\tau_{g}^{1} \mu_{5}+\mu_{3}^{\prime}\right)+f_{z} \mu_{4}^{\prime}+ \\
\left.f_{w}\left(\kappa_{n} \mu_{2}+\tau_{g}^{1} \mu_{3}+\tau_{g}^{2} \mu_{4}+\mu_{5}^{\prime}\right)\right)
\end{array}\right), \\
& \overline{\tau_{g}^{2}}=\overline{1} \mu_{11}^{2}\|\nabla f\|\left(\begin{array}{c}
-\left(f_{x} \mu_{7}+f_{y} \mu_{8}+f_{z} \mu_{9}+f_{w} \mu_{10}\right) \mu_{11}^{\prime}+\mu_{11}\left(f_{x} \mu_{7}^{\prime}\right. \\
-\kappa_{g}^{1} f_{x} \mu_{8}+\kappa_{g}^{2} f_{z} \mu_{8}-\kappa_{n} f_{x} \mu_{10}-\tau_{g}^{2} f_{z} \mu_{10}+ \\
f_{y}\left(\kappa_{g}^{1} \mu_{7}-\kappa_{g}^{2} \mu_{9}-\tau_{g}^{1} \mu_{10}+\mu_{8}^{\prime}\right)+ \\
\left.f_{z} \mu_{9}^{\prime}+f_{w}\left(\kappa_{n} \mu_{7}+\tau_{g}^{1} \mu_{8}+\tau_{g}^{2} \mu_{9}+\mu_{10}^{\prime}\right)\right)
\end{array}\right) .
\end{aligned}
$$

Proof. Since $\beta=\beta(s)$ is a TU-Smarandache curve reference to Frenet curve $r$. Then, by differentiating Eq. (3.5), we get

$$
\beta^{\prime}=\frac{1}{\sqrt{2}}\left(\left(\kappa_{g}^{1}-\kappa_{g}^{2}\right) \mathbf{P}+\left(\kappa_{n}+\tau_{g}^{2}\right) \mathbf{U}\right)
$$

Again, by differentiating Eq. (3.7), we have

$$
\beta^{\prime \prime}=\frac{1}{\sqrt{2}}\left(\begin{array}{c}
\left(-\kappa_{g}^{1}\left(\kappa_{g}^{1}-\kappa_{g}^{2}\right)-\kappa_{n}\left(\kappa_{n}+\tau_{g}^{2}\right)\right) \mathbf{T}-\tau_{g}^{1}\left(\kappa_{n}+\tau_{g}^{2}\right) \mathbf{P} \\
+\left(\left(\kappa_{g}^{1}-\kappa_{g}^{2}\right) \kappa_{g}^{2}-\left(\kappa_{n}+\tau_{g}^{2}\right) \tau_{g}^{2}\right) \mathbf{U}+\left(\kappa_{g}^{1}-\kappa_{g}^{2}\right) \tau_{g}^{1} \mathbf{N}
\end{array}\right) .
$$

Also, from Eq. (3.7), we obtain

$$
\overline{\mathbf{T}}=\frac{\left(\kappa_{g}^{1}-\kappa_{g}^{2}\right) \mathbf{P}+\left(\kappa_{n}+\tau_{g}^{2}\right) \mathbf{U}}{\mu_{1}},
$$

where

$$
\mu_{1}=\sqrt{2\left(\kappa_{g}^{1}-\kappa_{g}^{2}\right)^{2}+\left(\kappa_{n}+\tau_{g}^{2}\right)^{2}}
$$

and we have

$$
\begin{aligned}
& \overline{\mathbf{N}}=\frac{f_{x} \mathbf{T}+f_{y} \mathbf{P}+f_{z} \mathbf{U}+f_{w} \mathbf{N}}{\|\nabla f\|}, \\
& \overline{\mathbf{P}}=\frac{\mu_{2} \mathbf{T}+\mu_{3} \mathbf{P}+\mu_{4} \mathbf{U}+\mu_{5} \mathbf{N}}{\mu_{6}},
\end{aligned}
$$


where

$$
\begin{aligned}
& \mu_{2}=\frac{1}{\sqrt{2}}\left(\left(\left(\kappa_{g}^{1}\right)^{2}-\kappa_{g}^{1} \kappa_{g}^{2}+\kappa_{n}\left(\kappa_{n}+\tau_{g}^{2}\right)\right)\right)+\frac{\sigma_{2}}{\|\nabla f\|} f_{x}, \\
& \mu_{3}=\frac{1}{\sqrt{2}}\left(\tau_{g}^{1}\left(\kappa_{n}+\tau_{g}^{2}\right)\right)+\frac{\sigma_{2}}{\|\nabla f\|} f_{y}, \\
& \mu_{4}=\frac{1}{\sqrt{2}}\left(\left(\kappa_{g}^{2}\left(-\kappa_{g}^{1}+\kappa_{g}^{2}\right)+\tau_{g}^{2}\left(\kappa_{n}+\tau_{g}^{2}\right)\right)\right)+\frac{\sigma_{2}}{\|\nabla f\|} f_{z}, \\
& \mu_{5}= \frac{1}{\sqrt{2}}\left(\left(-\kappa_{g}^{1}+\kappa_{g}^{2}\right) \tau_{g}^{1}\right)+\frac{\sigma_{2}}{\|\nabla f\|} f_{w}, \\
& \sigma_{2}=\left\langle\beta^{\prime \prime}, \mathbf{N}\right\rangle=\frac{1}{\sqrt{2}\|\nabla f\|}\left(\begin{array}{c}
\left.\mu_{6}=\left(\kappa_{g}^{1}\right)^{2}+\kappa_{g}^{1} \kappa_{g}^{2}-\kappa_{n}\left(\kappa_{n}+\tau_{g}^{2}\right)\right) f_{x}+\tau_{g}^{1}\left(\kappa_{n}+\tau_{g}^{2}\right) f_{y} \\
+\left(\left(\kappa_{g}^{1}-\kappa_{g}^{2}\right) \kappa_{g}^{2}-\tau_{g}^{2}\left(\kappa_{n}+\tau_{g}^{2}\right)\right) f_{z}+\left(\kappa_{g}^{1}-\kappa_{g}^{2}\right) \tau_{g}^{1} f_{w}
\end{array}\right) .
\end{aligned}
$$

Also, we get

$$
\overline{\mathbf{U}}=\frac{\mu_{7} \mathbf{T}+\mu_{8} \mathbf{P}+\mu_{3} \mathbf{U}+\mu_{10} \mathbf{N}}{\delta}
$$

where

$$
\begin{aligned}
\mu_{7} & =-\kappa_{n} f_{z} \mu_{3}-\tau_{g}^{2} f_{z} \mu_{3}-\kappa_{g}^{1} f_{w} \mu_{4}+\kappa_{g}^{2} f_{w} \mu_{4}+\kappa_{n} f_{y} \mu_{4}+\tau_{g}^{2} f_{y} \mu_{4}+\kappa_{g}^{1} f_{z} \mu_{5}-\kappa_{g}^{2} f_{z} \mu_{5}, \\
\mu_{8} & =\kappa_{n} f_{z} \mu_{2}+\tau_{g}^{2} f_{z} \mu_{2}-\kappa_{n} f_{x} \mu_{4}-\tau_{g}^{2} f_{x} \mu_{4} \\
\mu_{9} & =\kappa_{g}^{1} f_{w} \mu_{2}-\kappa_{g}^{2} f_{w} \mu_{2}-\kappa_{n} f_{y} \mu_{2}-\tau_{g}^{2} f_{y} \mu_{2}+\kappa_{n} f_{x} \mu_{3}+\tau_{g}^{2} f_{x} \mu_{3}-\kappa_{g}^{1} f_{x} \mu_{5}+\kappa_{g}^{2} f_{x} \mu_{5}, \\
\mu_{10} & =-\kappa_{g}^{1} f_{z} \mu_{2}+\kappa_{g}^{2} f_{z} \mu_{2}+\kappa_{g}^{1} f_{x} \mu_{4}-\kappa_{g}^{2} f_{x} \mu_{4}, \\
\mu 11 & =\mu_{1} \mu_{6}\|\nabla f\| .
\end{aligned}
$$

In the light of the above calculations, the curvature functions $\overline{\kappa_{n}}, \overline{\kappa_{g}^{1}}, \overline{\kappa_{g}^{2}}, \overline{\tau_{g}^{1}}$ and $\overline{\tau_{g}^{2}}$ of $\beta$ are computed as in Eqs. (3.6).

Corollary 3.3. If $r$ is an asymptotic curve. Then, the following equations hold:

$$
\begin{aligned}
\overline{\mathbf{T}} & =\frac{\left(\kappa_{g}^{1}-\kappa_{g}^{2}\right) \mathbf{P}+\tau_{g}^{2} \mathbf{U}}{\mu_{1}}, \\
\overline{\mathbf{N}} & =\frac{f_{x} \mathbf{T}+f_{y} \mathbf{P}+f_{z} \mathbf{U}+f_{w} \mathbf{N}}{\|\nabla f\|} \\
\overline{\mathbf{P}} & =\frac{\mu_{13} \mathbf{T}+\mu_{14} \mathbf{P}+\mu_{15} \mathbf{U}+\mu_{16} \mathbf{N}}{\mu_{17}} \\
\overline{\mathbf{U}} & =\frac{\mu_{18} \mathbf{T}+\mu_{19} \mathbf{P}+\mu_{20} \mathbf{U}+\mu_{21} \mathbf{N}}{\mu_{22}}
\end{aligned}
$$


and the curvature functions are obtained as follows:

$$
\begin{aligned}
\overline{\kappa_{n}} & =\frac{\left(\kappa_{g}^{1}-\kappa_{g}^{2}\right) \tau_{g}^{1} f_{w}+\kappa_{g}^{1}\left(-\kappa_{g}^{1}+\kappa_{g}^{2}\right) f_{x}-\tau_{g}^{1} \tau_{g}^{2} f_{y}-\left(-\kappa_{g}^{1} \kappa_{g}^{2}+\left(\kappa_{g}^{2}\right)^{2}+\left(\tau_{g}^{2}\right)^{2}\right) f_{z}}{\mu_{12}\|\nabla f\|}, \\
\overline{\kappa_{g}^{1}} & =\frac{\kappa_{g}^{1}\left(-\kappa_{g}^{1}+\kappa_{g}^{2}\right) \mu_{13}+\left(\kappa_{g}^{1}-\kappa_{g}^{2}\right) \kappa_{g}^{2} \mu_{15}-\tau_{g}^{2}\left(\tau_{g}^{1} \mu_{14}+\tau_{g}^{2} \mu_{15}\right)+\left(\kappa_{g}^{1}-\kappa_{g}^{2}\right) \tau_{g}^{1} \mu_{16}}{\mu_{12} \mu_{17}}, \\
\overline{\kappa_{g}^{2}} & =\frac{1}{\mu_{17}^{2} \mu_{22}}\left(\begin{array}{c}
\mu_{17}\left(\kappa_{g}^{1} \mu_{13} \mu_{19}-\kappa_{g}^{2} \mu_{15} \mu_{19}-\tau_{g}^{1} \mu_{16} \mu_{19}-\tau_{g}^{2} \mu_{16} \mu_{20}+\right. \\
\tau_{g}^{2} \mu_{15} \mu_{21}+\mu_{14}\left(-\kappa_{g}^{1} \mu_{18}+\kappa_{g}^{2} \mu_{20}+\tau_{g}^{1} \mu_{21}\right)+\mu_{18} \mu_{13}^{\prime}+\mu_{19} \mu_{14}^{\prime}+ \\
\left.\mu_{20} \mu_{15}^{\prime}+\mu_{21} \mu_{16}^{\prime}\right)-\left(\mu_{13} \mu_{18}+\mu_{14} \mu_{19}+\mu_{15} \mu_{20}+\mu_{16} \mu_{21}\right) \mu_{17}^{\prime}
\end{array}\right), \\
\overline{\tau_{g}^{1}} & =\frac{1}{\mu_{17}^{2}\|\nabla f\|}\left(\begin{array}{c}
-f_{x}\left(\kappa_{g}^{1} \mu_{14} \mu_{17}-\mu_{17} \mu_{13}^{\prime}+\mu_{13} \mu_{17}^{\prime}\right)+f_{y}\left(\mu _ { 1 7 } \left(\kappa_{g}^{1} \mu_{13}-\right.\right. \\
\left.\left.\kappa_{g}^{2} \mu_{15}-\tau_{g}^{1} \mu_{16}+\mu_{14}^{\prime}\right)-\mu_{14} \mu_{17}^{\prime}\right)+f_{z}\left(\mu _ { 1 7 } \left(\kappa_{g}^{2} \mu_{14}-\tau_{g}^{2} \mu_{16}+\right.\right. \\
\left.\left.\mu_{15}^{\prime}\right)-\mu_{15} \mu_{17}^{\prime}\right)+f_{w}\left(\mu_{17}\left(\tau_{g}^{1} \mu_{14}+\tau_{g}^{2} \mu_{15}+\mu_{16}^{\prime}\right)-\mu_{16} \mu_{17}^{\prime}\right)
\end{array}\right), \\
\overline{\tau_{g}^{2}}= & \frac{1}{\mu_{22}^{2}\|\nabla f\|}\left(\begin{array}{c}
-f_{x}\left(\kappa_{g}^{1} \mu_{19} \mu_{22}-\mu_{22} \mu_{18}^{\prime}+\mu_{18} \mu_{22}^{\prime}\right)+f_{y}\left(\mu _ { 2 2 } \left(\kappa_{g}^{1} \mu_{18}-\kappa_{g}^{2} \mu_{20}-\right.\right. \\
\left.\left.\tau_{g}^{1} \mu_{21}+\mu_{19}^{\prime}\right)-\mu_{19} \mu_{22}^{\prime}\right)+f_{z}\left(\mu_{22}\left(\kappa_{g}^{2} \mu_{19}-\tau_{g}^{2} \mu_{21}+\mu_{20}^{\prime}\right)-\right. \\
\left.\mu_{20} \mu_{22}^{\prime}\right)+f_{w}\left(\mu_{22}\left(\tau_{g}^{1} \mu_{19}+\tau_{g}^{2} \mu_{20}+\mu_{21}^{\prime}\right)-\mu_{21} \mu_{22}^{\prime}\right)
\end{array}\right),
\end{aligned}
$$

where

$$
\begin{aligned}
& \mu_{12}=\sqrt{\left(\kappa_{g}^{1}-\kappa_{g}^{2}\right)^{2}+\left(\tau_{g}^{2}\right)^{2}}, \\
& \mu_{13}=\sqrt{2}\left(\left(\kappa_{g}^{1}\right)^{2}-\kappa_{g}^{1} \kappa_{g}^{2}\right)\|\nabla f\|+2 \sigma_{2} f_{x} \\
& \mu_{14}=\sqrt{2} \tau_{g}^{1} \tau_{g}^{2}\|\nabla f\|+2 \sigma_{2} f_{y} \\
& \mu_{15}=\sqrt{2}\left(\kappa_{g}^{2}\left(-\kappa_{g}^{1}+\kappa_{g}^{2}\right)+\left(\tau_{g}^{2}\right)^{2}\right)\|\nabla f\|+2 \sigma_{2} f_{z}, \\
& \mu_{16}=\sqrt{2}\left(-\kappa_{g}^{1}+\kappa_{g}^{2}\right) \tau_{g}^{1}\|\nabla f\|+2 \sigma_{2} f_{w}, \\
& \mu_{17}^{2}=\left(\mu_{13}\right)^{2}+\left(\mu_{14}\right)^{2}+\left(\mu_{15}\right)^{2}+\left(\mu_{16}\right)^{2}, \\
& \mu_{18}=-\left(\kappa_{g}^{1} \mu_{15} f_{w}-\kappa_{g}^{2} \mu_{15} f_{w}-\tau_{g}^{2} \mu_{15} f_{y}+\tau_{g}^{2} \mu_{14} f_{z}-\kappa_{g}^{1} \mu_{16} f_{z}+\kappa_{g}^{2} \mu_{16} f_{z}\right), \\
& \mu_{19}=-\left(\tau_{g}^{2} \mu_{15} f_{x}-\tau_{g}^{2} \mu_{13} f_{z}\right), \\
& \mu_{20}=-\left(-\kappa_{g}^{1} \mu_{13} f_{w}+\kappa_{g}^{2} \mu_{13} f_{w}-\tau_{g}^{2} \mu_{14} f_{x}+\kappa_{g}^{1} \mu_{16} f_{x}-\kappa_{g}^{2} \mu_{16} f_{x}+\tau_{g}^{2} \mu_{13} f_{y}\right), \\
& \mu_{21}=-\left(-\kappa_{g}^{1} \mu_{15} f_{x}+\kappa_{g}^{2} \mu_{15} f_{x}+\kappa_{g}^{1} \mu_{13} f_{z}-\kappa_{g}^{2} \mu_{13} f_{z}\right), \\
& \mu_{22}=\mu_{12} \mu_{17}\|\nabla f\| .
\end{aligned}
$$


Corollary 3.4. If $r$ is a line of curvature curve. Then, the following equations hold:

$$
\begin{aligned}
\overline{\mathbf{T}} & =\frac{\left(\kappa_{g}^{1}-\kappa_{g}^{2}\right) \mathbf{P}+\kappa_{n} \mathbf{N}}{\mu_{23}}, \\
\overline{\mathbf{N}} & =\frac{f_{x} \mathbf{T}+f_{y} \mathbf{P}+f_{z} \mathbf{U}+f_{w} \mathbf{N}}{\|\nabla f\|}, \\
\overline{\mathbf{P}} & =\frac{\mu_{24} \mathbf{T}+\mu_{25} \mathbf{P}+\mu_{26} \mathbf{U}+\mu_{27} \mathbf{N}}{\mu_{28}}, \\
\overline{\mathbf{U}} & =\frac{\mu_{29} \mathbf{T}+\mu_{30} \mathbf{P}+\mu_{31} \mathbf{U}+\mu_{32} \mathbf{N}}{\mu_{33}},
\end{aligned}
$$

and the curvature functions are obtained as follows:

$$
\begin{aligned}
& \overline{\kappa_{n}}=\frac{-\left(\left(\kappa_{g}^{1}\right)^{2}-\kappa_{g}^{1} \kappa_{g}^{2}+\kappa_{n}^{2}\right) f_{x}+\left(\kappa_{g}^{1}-\kappa_{g}^{2}\right) \kappa_{g}^{2} f_{z}}{\mu_{23}\|\nabla f\|}, \\
& \bar{\kappa}_{g}^{1}=\frac{-\left(\left(\kappa_{g}^{1}\right)^{2}-\kappa_{g}^{1} \kappa_{g}^{2}+\kappa_{n}^{2}\right) \mu_{24}+\left(\kappa_{g}^{1}-\kappa_{g}^{2}\right) \kappa_{g}^{2} \mu_{26}}{\mu_{23} \mu_{28}}, \\
& \overline{\kappa_{g}^{2}}=\overline{\mu_{17}^{2} \mu_{22}}\left(\begin{array}{c}
\mu_{28}\left(-\kappa_{n} \mu_{27} \mu_{29}+\mu_{25}\left(-\kappa_{g}^{1} \mu_{29}+\kappa_{g}^{2} \mu_{31}\right)+\mu_{29} \mu_{24}^{\prime}+\right. \\
\mu_{30}\left(\kappa_{g}^{1} \mu_{24}-\kappa_{g}^{2} \mu_{26}+\mu_{25}^{\prime}\right)+\mu_{31} \mu_{26}^{\prime}+\mu_{32}\left(\kappa_{n} \mu_{24}+\right. \\
\left.\left.\mu_{27}^{\prime}\right)\right)-\left(\mu_{24} \mu_{29}+\mu_{25} \mu_{30}+\mu_{26} \mu_{31}+\mu_{27} \mu_{32}\right) \mu_{28}^{\prime}
\end{array}\right), \\
& \overline{\tau_{g}^{1}}=\frac{1}{\mu_{28}^{2}\|\nabla f\|}\left(\begin{array}{c}
-f_{x}\left(\mu_{28}\left(\kappa_{g}^{1} \mu_{25}+\kappa_{n} \mu_{27}-\mu_{24}^{\prime}\right)+\mu_{24} \mu_{28}^{\prime}\right)+ \\
f_{y}\left(\mu_{28}\left(\kappa_{g}^{1} \mu_{24}-\kappa_{g}^{2} \mu_{26}+\mu_{25}^{\prime}\right)-\mu_{25} \mu_{28}^{\prime}\right)+ \\
f_{z}\left(\mu_{28}\left(\kappa_{g}^{2} \mu_{25}+\mu_{26}^{\prime}\right)-\mu_{26} \mu_{28}^{\prime}\right)+ \\
f_{w}\left(\mu_{28}\left(\kappa_{n} \mu_{24}+\mu_{27}^{\prime}\right)-\mu_{27} \mu_{28}^{\prime}\right)
\end{array}\right) \text {, } \\
& \overline{\tau_{g}^{2}}=\frac{1}{\mu_{33}^{2}\|\nabla f\|}\left(\begin{array}{c}
-f_{x}\left(\mu_{33}\left(\kappa_{g}^{1} \mu_{30}+\kappa_{n} \mu_{32}-\mu_{29}^{\prime}\right)+\mu_{29} \mu_{33}^{\prime}\right)+ \\
f_{y}\left(\mu_{33}\left(\kappa_{g}^{1} \mu_{29}-\kappa_{g}^{2} \mu_{31}+\mu_{30}^{\prime}\right)-\mu_{30} \mu_{33}^{\prime}\right)+ \\
f_{z}\left(\mu_{33}\left(\kappa_{g}^{2} \mu_{30}+\mu_{31}^{\prime}\right)-\mu_{31} \mu_{33}^{\prime}\right)+ \\
f_{w}\left(\mu_{33}\left(\kappa_{n} \mu_{29}+\mu_{32}^{\prime}\right)-\mu_{32} \mu_{33}^{\prime}\right)
\end{array}\right) \text {, }
\end{aligned}
$$

where

$$
\begin{aligned}
& \mu_{23}=\sqrt{\left(\kappa_{g}^{1}-\kappa_{g}^{2}\right)^{2}+\kappa_{n}^{2}}, \mu_{24}=\sqrt{2}\left(\left(\kappa_{g}^{1}\right)^{2}-\kappa_{g}^{1} \kappa_{g}^{2}+\kappa_{n}^{2}\right)\|\nabla f\|+2 \sigma_{2} f_{x}, \\
& \mu_{25}=2 \sigma_{2} f_{y}, \mu_{26}=\sqrt{2} \kappa_{g}^{2}\left(-\kappa_{g}^{1}+\kappa_{g}^{2}\right)\|\nabla f\|+2 \sigma_{2} f_{z}, \\
& \mu_{27}=2 \sigma_{2} f_{w}, \mu_{28}^{2}=\left(\mu_{24}\right)^{2}+\left(\mu_{25}\right)^{2}+\left(\mu_{26}\right)^{2}+\left(\mu_{27}\right)^{2}, \\
& \mu_{29}=-\left(\kappa_{g}^{1} \mu_{26} f_{w}-\kappa_{g}^{2} \mu_{26} f_{w}-\kappa_{n} \mu_{26} f_{y}+\kappa_{n} \mu_{25} f_{z}-\kappa_{g}^{1} \mu_{27} f_{z}+\kappa_{g}^{2} \mu_{27} f_{z}\right), \\
& \mu_{30}=-\left(\kappa_{n} \mu_{26} f_{x}-\kappa_{n} \mu_{25} f_{z}\right), \\
& \mu_{31}=-\left(-\kappa_{g}^{1} \mu_{24} f_{w}+\kappa_{g}^{2} \mu_{24} f_{w}-\kappa_{n} \mu_{25} f_{x}+\kappa_{g}^{1} \mu_{27} f_{x}-\kappa_{g}^{2} \mu_{27} f_{x}+\kappa_{n} \mu_{24} f_{y}\right), \\
& \mu_{32}=-\left(-\kappa_{g}^{1} \mu_{26} f_{x}+\kappa_{g}^{2} \mu_{26} f_{x}+\kappa_{g}^{1} \mu_{24} f_{z}-\kappa_{g}^{2} \mu_{24} f_{z}\right), \\
& \mu_{33}=\mu_{24} \mu_{28}\|\nabla f\| .
\end{aligned}
$$




\subsection{PU-Smarandache curves.}

Definition 3.4. Let $M$ be an oriented hypersurface in $\mathbb{E}^{4}$ and the Frenet curve $\gamma=\gamma(s)$ lying fully on $M$ with Darboux frame $\{\mathbf{T}, \mathbf{P}, \mathbf{U}, \mathbf{N}\}$ and non-zero curvatures $\kappa_{n}, \kappa_{g}^{1}, \kappa_{g}^{2}, \tau_{g}^{1}$ and $\tau_{g}^{2}$. Then the $\boldsymbol{P} \boldsymbol{U}$-Smarandache curve of $\gamma$ is defined as

$$
\gamma(u)=\frac{1}{\sqrt{2}}(\mathbf{P}+\mathbf{U})
$$

Theorem 3.3. Let $r=r(u)$ be a Frenet curve lying on a hypersurface $M$ in $\mathbb{E}^{4}$ with Darboux frame $\{\mathbf{T}, \mathbf{P}, \mathbf{U}, \mathbf{N}\}$ and non-zero curvatures $\kappa_{n}, \kappa_{g}^{1}, \kappa_{g}^{2}, \tau_{g}^{1}$ and $\tau_{g}^{2}$. Then the curvature functions of the $\mathbf{P} \mathbf{U}-$ Smarandache curve of $r$ satisfy the following equations:

$$
\begin{aligned}
& \overline{\kappa_{n}}=\frac{-1}{\nu_{1}\|\nabla f\|}\left(\begin{array}{c}
\left(\kappa_{g}^{1} \kappa_{n}+\kappa_{g}^{2}\left(\tau_{g}^{1}-\tau_{g}^{2}\right)\right) f_{w}+\left(-\kappa_{g}^{1} \kappa_{g}^{2}+\right. \\
\left.\kappa_{n}\left(\tau_{g}^{1}+\tau_{g}^{2}\right)\right) f_{x}+\left(\left(\kappa_{g}^{1}\right)^{2}+\left(\kappa_{g}^{2}\right)^{2}+\tau_{g}^{1}\left(\tau_{g}^{1}+\right.\right. \\
\left.\left.\tau_{g}^{2}\right)\right) f_{y}+\left(\left(\kappa_{g}^{2}\right)^{2}+\tau_{g}^{2}\left(\tau_{g}^{1}+\tau_{g}^{2}\right)\right) f_{z}
\end{array}\right) \\
& \overline{\kappa_{g}^{1}}=\frac{1}{\nu_{1} \nu_{6}}\left(\begin{array}{c}
\left(\kappa_{g}^{1} \kappa_{n}+\kappa_{g}^{2}\left(\tau_{g}^{1}-\tau_{g}^{2}\right)\right) \nu_{5}+\left(-\kappa_{g}^{1} \kappa_{g}^{2}+\right. \\
\left.\kappa_{n}\left(\tau_{g}^{1}+\tau_{g}^{2}\right)\right) \nu_{2}+\left(\left(\kappa_{g}^{1}\right)^{2}+\left(\kappa_{g}^{2}\right)^{2}+\tau_{g}^{1}\left(\tau_{g}^{1}+\right.\right. \\
\left.\left.\tau_{g}^{2}\right)\right) \nu_{3}+\left(\left(\kappa_{g}^{2}\right)^{2}+\tau_{g}^{2}\left(\tau_{g}^{1}+\tau_{g}^{2}\right)\right) \nu_{4}
\end{array}\right) \\
& \overline{\kappa_{g}^{2}}=\frac{1}{\nu_{6}^{2} \nu_{11}}\left(\begin{array}{c}
-\left(\nu_{2} \nu_{7}+\nu_{3} \nu_{8}+\nu_{4} \nu_{9}+\nu_{5} \nu_{10}\right) \nu_{6}^{\prime}+\nu_{6}\left(\left(\kappa_{g}^{1} \nu_{2}-\kappa_{g}^{2} \nu_{4}\right) \nu_{8}\right. \\
-\nu_{5}\left(\kappa_{n} \nu_{7}+\tau_{g}^{1} \nu_{8}+\tau_{g}^{2} \nu_{9}\right)+\nu_{3}\left(-\kappa_{g}^{1} \nu_{7}+\kappa_{g}^{2} \nu_{9}+\tau_{g}^{1} \nu_{10}\right)+ \\
\left.\nu_{7} \nu_{2}^{\prime}+\nu_{8} \nu_{3}^{\prime}+\nu_{9} \nu_{4}^{\prime}+\nu_{10}\left(\kappa_{n} \nu_{2}+\tau_{g}^{2} \nu_{4}+\nu_{5}^{\prime}\right)\right)
\end{array}\right), \\
& \overline{\tau_{g}^{1}}=\frac{1}{\nu_{6}^{2}\|\nabla f\|}\left(\begin{array}{c}
-\left(f_{x} \nu_{2}+f_{y} \nu_{3}+f_{z} \nu_{4}+f_{w} \nu_{5}\right) \nu_{6}^{\prime}+\nu_{6}\left(-\kappa_{g}^{1} f_{x} \nu_{3}+\kappa_{g}^{2} f_{z} \nu_{3}\right. \\
-\kappa_{n} f_{x} \nu_{5}-\tau_{g}^{2} f_{z} \nu_{5}+f_{x} \nu_{2}^{\prime}+f_{y}\left(\kappa_{g}^{1} \nu_{2}-\kappa_{g}^{2} \nu_{4}-\tau_{g}^{1} \nu_{5}\right. \\
\left.\left.+\nu_{3}^{\prime}\right)+f_{z} \nu_{4}^{\prime}+f_{w}\left(\kappa_{n} \nu_{2}+\tau_{g}^{1} \nu_{3}+\tau_{g}^{2} \nu_{4}+\nu_{5}^{\prime}\right)\right)
\end{array}\right), \\
& \overline{\tau_{g}^{2}}=\frac{1}{\nu_{11}^{2}\|\nabla f\|}\left(\begin{array}{c}
-\left(f_{x} \nu_{7}+f_{y} \nu_{8}+f_{z} \nu_{9}+f_{w} \nu_{10}\right) \nu_{11}^{\prime}+\nu_{11}\left(-\kappa_{g}^{1} f_{x} \nu_{8}+\kappa_{g}^{2} f_{z} \nu_{8}\right. \\
-\kappa_{n} f_{x} \nu_{10}-\tau_{g}^{2} f_{z} \nu_{10}+f_{x} \nu_{7}^{\prime}+f_{y}\left(\kappa_{g}^{1} \nu_{7}-\kappa_{g}^{2} \nu_{9}-\tau_{g}^{1} \nu_{10}\right. \\
\left.\left.+\nu_{8}^{\prime}\right)+f_{z} \nu_{9}^{\prime}+f_{w}\left(\kappa_{n} \nu_{7}+\tau_{g}^{1} \nu_{8}+\tau_{g}^{2} \nu_{9}+\nu_{10}^{\prime}\right)\right)
\end{array}\right) .
\end{aligned}
$$

Proof. Since $\gamma=\gamma(s)$ is a PU- Smarandache curve reference to Frenet curve $r$. Then, by differentiating Eq. (3.8), we get

$$
\begin{aligned}
\gamma^{\prime}= & \frac{-\kappa_{g}^{1} \mathbf{T}+\kappa_{g}^{2} \mathbf{P}+\kappa_{g}^{2} \mathbf{U}+\left(\tau_{g}^{1}+\tau_{g}^{2}\right) \mathbf{N}}{\sqrt{2}}, \\
\gamma^{\prime \prime}= & \frac{1}{\sqrt{2}}\left(\kappa_{g}^{1} \kappa_{g}^{2}-\kappa_{n}\left(\tau_{g}^{1}+\tau_{g}^{2}\right)\right) \mathbf{T}+\left(-\left(\kappa_{g}^{1}\right)^{2}-\left(\kappa_{g}^{2}\right)^{2}-\tau_{g}^{1}\left(\tau_{g}^{1}+\tau_{g}^{2}\right)\right) \mathbf{P} \\
& +\left(-\left(\kappa_{g}^{2}\right)^{2}-\tau_{g}^{2}\left(\tau_{g}^{1}+\tau_{g}^{2}\right)\right) \mathbf{U}+\left(-\kappa_{g}^{1} \kappa_{n}-\kappa_{g}^{2} \tau_{g}^{1}+\kappa_{g}^{2} \tau_{g}^{2}\right) \mathbf{N}
\end{aligned}
$$


Using Eqs. (3.10), we have

$$
\begin{aligned}
& \overline{\mathbf{T}}=\frac{-\kappa_{g}^{1} \mathbf{T}+\kappa_{g}^{2} \mathbf{P}+\kappa_{g}^{2} \mathbf{U}+\left(\tau_{g}^{1}+\tau_{g}^{2}\right) \mathbf{N}}{\nu_{1}} \\
& \overline{\mathbf{N}}=\frac{f_{x} \mathbf{T}+f_{y} \mathbf{P}+f_{z} \mathbf{U}+f_{w} \mathbf{N}}{\|\nabla f\|}
\end{aligned}
$$

where

$$
\nu_{1}=\sqrt{2\left(\kappa_{g}^{2}\right)^{2}+\left(\tau_{g}^{1}+\tau_{g}^{2}\right)^{2}+\left(\kappa_{g}^{1}\right)^{2}}
$$

On the other hand, we get

$$
\overline{\mathbf{P}}=\frac{\nu_{2} \mathbf{T}+\nu_{3} \mathbf{P}+\nu_{4} \mathbf{U}+\nu_{5} \mathbf{N}}{\nu_{6}}
$$

where

$$
\begin{aligned}
& \nu_{2}=\sqrt{2}\left(-\kappa_{g}^{1} \kappa_{g}^{2}+\kappa_{n}\left(\tau_{g}^{1}+\tau_{g}^{2}\right)\right)\|\nabla f\|+2 \sigma_{3} f_{x} \\
& \nu_{3}=\sqrt{2}\left(\left(\kappa_{g}^{1}\right)^{2}+\left(\kappa_{g}^{2}\right)^{2}+\tau_{g}^{1}\left(\tau_{g}^{1}+\tau_{g}^{2}\right)\right)\|\nabla f\|+2 \sigma_{3} f_{y} \\
& \nu_{4}=\sqrt{2}\left(\left(\kappa_{g}^{2}\right)^{2}+\tau_{g}^{2}\left(\tau_{g}^{1}+\tau_{g}^{2}\right)\right)\|\nabla f\|+2 \sigma_{3} f_{z} \\
& \nu_{5}=\sqrt{2}\left(\kappa_{g}^{1} \kappa_{n}+\kappa_{g}^{2} \tau_{g}^{1}-\kappa_{g}^{2} \tau_{g}^{2}\right)\|\nabla f\|+2 \sigma_{3} f_{w} \\
& \nu_{6}^{2}=\nu_{2}^{2}+\nu_{3}^{2}+\nu_{4}^{2}+\nu_{5}^{2}, \\
& \sigma_{3}=\left\langle\gamma^{\prime \prime}, \mathbf{N}\right\rangle=\frac{1}{2\|\nabla f\|}\left(\begin{array}{c}
-\left(\kappa_{g}^{1} \kappa_{n}+\kappa_{g}^{2}\left(\tau_{g}^{1}-\tau_{g}^{2}\right)\right) f_{w}+\left(\kappa_{g}^{1} \kappa_{g}^{2}-\right. \\
\left.\kappa_{n}\left(\tau_{g}^{1}+\tau_{g}^{2}\right)\right) f_{x}-\left(\left(\kappa_{g}^{1}\right)^{2}+\left(\kappa_{g}^{2}\right)^{2}+\tau_{g}^{1}\left(\tau_{g}^{1}+\right.\right. \\
\left.\left.\tau_{g}^{2}\right)\right) f_{y}-\left(\left(\kappa_{g}^{2}\right)^{2}+\tau_{g}^{2}\left(\tau_{g}^{1}+\tau_{g}^{2}\right)\right) f_{z}
\end{array}\right) .
\end{aligned}
$$

Also, we get

$$
\overline{\mathbf{U}}=\frac{\nu_{7} \mathbf{T}+\nu_{8} \mathbf{P}+\nu_{9} \mathbf{U}+\nu_{10} \mathbf{N}}{\nu_{11}}
$$

where

$$
\begin{aligned}
\nu_{7} & =\kappa_{g}^{2} f_{w} \nu_{3}-\tau_{g}^{1} f_{z} \nu_{3}-\tau_{g}^{2} f_{z} \nu_{3}+\kappa_{g}^{2} f_{w} \nu_{4}+\tau_{g}^{1} f_{y} \nu_{4}+\tau_{g}^{2} f_{y} \nu_{4}-\kappa_{g}^{2}\left(f_{y}+f_{z}\right) \nu_{5}, \\
\nu_{8} & =-f_{w}\left(\kappa_{g}^{2} \nu_{2}+\kappa_{g}^{1} \nu_{4}\right)+f_{z}\left(\left(\tau_{g}^{1}+\tau_{g}^{2}\right) \nu_{2}+\kappa_{g}^{1} \nu_{5}\right)+f_{x}\left(-\left(\tau_{g}^{1}+\tau_{g}^{2}\right) \nu_{4}+\kappa_{g}^{2} \nu_{5}\right), \\
\nu_{9} & =f_{w}\left(-\kappa_{g}^{2} \nu_{2}+\kappa_{g}^{1} \nu_{3}\right)-f_{y}\left(\left(\tau_{g}^{1}+\tau_{g}^{2}\right) \nu_{2}+\kappa_{g}^{1} \nu_{5}\right)+f_{x}\left(\left(\tau_{g}^{1}+\tau_{g}^{2}\right) \nu_{3}+\kappa_{g}^{2} \nu_{5}\right), \\
\nu_{10} & =\kappa_{g}^{2} f_{y} \nu_{2}+\kappa_{g}^{2} f_{z} \nu_{2}-\kappa_{g}^{2} f_{x} \nu_{3}-\kappa_{g}^{1} f_{z} \nu_{3}-\kappa_{g}^{2} f_{x} \nu_{4}+\kappa_{g}^{1} f_{y} \nu_{4}, \\
\nu_{11} & =\nu_{1}\|\nabla f\| \nu_{6} .
\end{aligned}
$$

In the light of the above calculations, the curvature functions $\overline{\kappa_{n}}, \overline{\kappa_{g}^{1}}, \overline{\kappa_{g}^{2}}, \overline{\tau_{g}^{1}}$ and $\overline{\tau_{g}^{2}}$ of $\gamma$ are computed as in Eqs. (3.9). 
Corollary 3.5. If $r$ is an asymptotic curve. Then, the following equations hold:

$$
\begin{aligned}
& \overline{\mathbf{T}}=\frac{-\kappa_{g}^{1} \mathbf{T}-\kappa_{g}^{2} \mathbf{P}+\kappa_{g}^{2} \mathbf{U}+\left(\tau_{g}^{1}+\tau_{g}^{2}\right) \mathbf{N}}{\nu_{12}}, \\
& \overline{\mathbf{N}}=\frac{f_{x} \mathbf{T}+f_{y} \mathbf{P}+f_{z} \mathbf{U}+f_{w} \mathbf{N}}{\|\nabla f\|}, \\
& \overline{\mathbf{P}}=\frac{\nu_{13} \mathbf{T}+\nu_{14} \mathbf{P}+\nu_{15} \mathbf{U}+\nu_{16} \mathbf{N}}{\nu_{17}}, \\
& \overline{\mathbf{U}}=\frac{\nu_{18} \mathbf{T}+\nu_{19} \mathbf{P}+\nu_{20} \mathbf{U}+\nu_{21} \mathbf{N}}{\nu_{22}}
\end{aligned}
$$

and the curvature functions are obtained as follows:

$$
\begin{aligned}
& \overline{\kappa_{n}}=\frac{1}{\nu_{17}\|\nabla f\|}\left(\begin{array}{c}
\kappa_{g}^{2}\left(-\tau_{g}^{1}+\tau_{g}^{2}\right) f_{w}+\kappa_{g}^{1} \kappa_{g}^{2} f_{x}-\left(\left(\kappa_{g}^{1}\right)^{2}+\left(\kappa_{g}^{2}\right)^{2}+\right. \\
\left.\tau_{g}^{1}\left(\tau_{g}^{1}+\tau_{g}^{2}\right)\right) f_{y}-\left(\left(\kappa_{g}^{2}\right)^{2}+\tau_{g}^{2}\left(\tau_{g}^{1}+\tau_{g}^{2}\right)\right) f_{z}
\end{array}\right), \\
& \overline{\kappa_{g}^{1}}=\frac{1}{\nu_{12} \nu_{17}}\left(\begin{array}{c}
\kappa_{g}^{1} \kappa_{g}^{2} \nu_{13}-\left(\left(\kappa_{g}^{1}\right)^{2}+\left(\kappa_{g}^{2}\right)^{2}+\tau_{g}^{1}\left(\tau_{g}^{1}+\tau_{g}^{2}\right)\right) \nu_{14}- \\
\left(\left(\kappa_{g}^{2}\right)^{2}+\tau_{g}^{2}\left(\tau_{g}^{1}+\tau_{g}^{2}\right)\right) \nu_{15}+\kappa_{g}^{2}\left(-\tau_{g}^{1}+\tau_{g}^{2}\right) \nu_{16}
\end{array}\right), \\
& \overline{\kappa_{g}^{2}}=\frac{1}{\nu_{17}^{2} \nu_{22}}\left(\begin{array}{c}
\nu_{17}\left(\kappa_{g}^{1} \nu_{13} \nu_{19}-\kappa_{g}^{2} \nu_{15} \nu_{19}-\tau_{g}^{1} \nu_{16} \nu_{19}-\tau_{g}^{2} \nu_{16} \nu_{20}+\right. \\
\tau_{g}^{2} \nu_{15} \nu_{21}+\nu_{14}\left(-\kappa_{g}^{1} \nu_{18}+\kappa_{g}^{2} \nu_{20}+\tau_{g}^{1} \nu_{21}\right)+\nu_{18} \nu_{13}^{\prime}+\nu_{19} \nu_{14}^{\prime}+ \\
\left.\nu_{20} \nu_{15}^{\prime}+\nu_{21} \nu_{16}^{\prime}\right)-\left(\nu_{13} \nu_{18}+\nu_{14} \nu_{19}+\nu_{15} \nu_{20}+\nu_{16} \nu_{21}\right) \nu_{17}^{\prime}
\end{array}\right), \\
& \overline{\tau_{g}^{1}=} \frac{1}{\nu_{17}^{2}\|\nabla f\|}\left(\begin{array}{c}
-f_{x}\left(\kappa_{g}^{1} \nu_{14} \nu_{17}-\nu_{17} \nu_{13}^{\prime}+\nu_{13} \nu_{17}^{\prime}\right)+f_{y}\left(\nu _ { 1 7 } \left(\kappa_{g}^{1} \nu_{13}-\kappa_{g}^{2} \nu_{15}-\right.\right. \\
\left.\left.\nu_{16}+\nu_{14}^{\prime}\right)-\nu_{14} \nu_{17}^{\prime}\right)+f_{z}\left(\nu_{17}\left(\kappa_{g}^{2} \nu_{14}-\tau_{g}^{2} \nu_{16}+\nu_{15}^{\prime}\right)-\right. \\
\nu_{w}\left(\nu_{17}\left(\tau_{g}^{1} \nu_{14}+\tau_{g}^{2} \nu_{15}+\nu_{16}^{\prime}\right)-\nu_{16} \nu_{17}^{\prime}\right) \\
-f_{x}\left(\kappa_{g}^{1} \nu_{19} \nu_{22}-\nu_{22} \nu_{18}^{\prime}+\nu_{18} \nu_{22}^{\prime}\right)+f_{y}\left(\nu _ { 2 2 } \left(\kappa_{g}^{1} \nu_{18}-\right.\right. \\
\left.\left.\kappa_{g}^{2} \nu_{20}-\tau_{g}^{1} \nu_{21}+\nu_{19}^{\prime}\right)-\nu_{19} \nu_{22}^{\prime}\right)+f_{z}\left(\nu _ { 2 2 } \left(\kappa_{g}^{2} \nu_{19}-\tau_{g}^{2} \nu_{21}+\right.\right. \\
\left.\left.\nu_{20}^{\prime}\right)-\nu_{20} \nu_{22}^{\prime}\right)+f_{w}\left(\nu_{22}\left(\tau_{g}^{1} \nu_{19}+\tau_{g}^{2} \nu_{20}+\nu_{21}^{\prime}\right)-\nu_{21} \nu_{22}^{\prime}\right)
\end{array}\right),
\end{aligned}
$$

where

$$
\begin{aligned}
& \nu_{12}=\sqrt{2\left(\kappa_{g}^{2}\right)^{2}+\left(\tau_{g}^{1}+\tau_{g}^{2}\right)^{2}+\left(\kappa_{g}^{1}\right)^{2}}, \nu_{13}=\sqrt{2}\left(-\kappa_{g}^{1} \kappa_{g}^{2}\right)\|\nabla f\|+2 \sigma_{3} f_{x}, \\
& \nu_{14}=\sqrt{2}\left(\left(\kappa_{g}^{1}\right)^{2}+\left(\kappa_{g}^{2}\right)^{2}+\tau_{g}^{1}\left(\tau_{g}^{1}+\tau_{g}^{2}\right)\right)\|\nabla f\|+2 \sigma_{3} f_{y}, \\
& \nu_{15}=\sqrt{2}\left(\left(\kappa_{g}^{2}\right)^{2}+\tau_{g}^{2}\left(\tau_{g}^{1}+\tau_{g}^{2}\right)\right)\|\nabla f\|+2 \sigma_{3} f_{z}, \\
& \nu_{16}=\sqrt{2}\left(\kappa_{g}^{2} \tau_{g}^{1}-\kappa_{g}^{2} \tau_{g}^{2}\right)\|\nabla f\|+2 \sigma_{3} f_{w}, \nu_{17}^{2}=\nu_{12}^{2}+\nu_{13}^{2}+\nu_{14}^{2}+\nu_{15}^{2}, \\
& \nu_{18}=-\kappa_{g}^{2} \nu_{16} f_{y}+\nu_{15}\left(\kappa_{g}^{2} f_{w}+\left(\tau_{g}^{1}+\tau_{g}^{2}\right) f_{y}\right)-\kappa_{g}^{2} \nu_{16} f_{z}+\nu_{14}\left(\kappa_{g}^{2} f_{w}-\left(\tau_{g}^{1}+\tau_{g}^{2}\right) f_{z}\right), \\
& \nu_{19}=-\kappa_{g}^{2} \nu_{13} f_{w}+\kappa_{g}^{2} \nu_{16} f_{x}-\nu_{15}\left(\kappa_{g}^{1} f_{w}+\left(\tau_{g}^{1}+\tau_{g}^{2}\right) f_{x}\right)+\left(\tau_{g}^{1}+\tau_{g}^{2}\right) \nu_{13} f_{z}+\kappa_{g}^{1} \nu_{16} f_{z}, \\
& \nu_{20}=-\kappa_{g}^{2} \nu_{13} f_{w}+\kappa_{g}^{2} \nu_{16} f_{x}+\nu_{14}\left(\kappa_{g}^{1} f_{w}+\left(\tau_{g}^{1}+\tau_{g}^{2}\right) f_{x}\right)-\tau_{g}^{1} \nu_{13} f_{y}-\tau_{g}^{2} \nu_{13} f_{y}-\kappa_{g}^{1} \nu_{16} f_{y}, \\
& \nu_{21}=\kappa_{g}^{2} \nu_{13} f_{y}+\nu_{15}\left(-\kappa_{g}^{2} f_{x}+\kappa_{g}^{1} f_{y}\right)+\kappa_{g}^{2} \nu_{13} f_{z}-\nu_{14}\left(\kappa_{g}^{2} f_{x}+\kappa_{g}^{1} f_{z}\right), \\
& \nu_{22}=\nu_{12} \nu_{17}\|\nabla f\| .
\end{aligned}
$$


Corollary 3.6. If $r$ is a line of curvature. Then, the following equations hold:

$$
\begin{aligned}
\overline{\mathbf{T}} & =-\frac{\kappa_{g}^{1} \mathbf{T}+\kappa_{g}^{2} \mathbf{P}-\kappa_{g}^{2} \mathbf{U}}{\nu_{12}}, \\
\overline{\mathbf{N}} & =\frac{f_{x} \mathbf{T}+f_{y} \mathbf{P}+f_{z} \mathbf{U}+f_{w} \mathbf{N}}{\|\nabla f\|}, \\
\overline{\mathbf{P}} & =\frac{\nu_{13} \mathbf{T}+\nu_{14} \mathbf{P}+\nu_{15} \mathbf{U}+\nu_{16} \mathbf{N}}{\nu_{17}}, \\
\overline{\mathbf{U}} & =\frac{\nu_{18} \mathbf{T}+\nu_{19} \mathbf{P}+\nu_{20} \mathbf{U}+\nu_{21} \mathbf{N}}{\nu_{22}},
\end{aligned}
$$

and the curvature functions are obtained as follows:

$$
\begin{aligned}
\overline{\kappa_{n}} & =\frac{-1}{\nu_{23}\|\nabla f\|}\left(\kappa_{g}^{1} \kappa_{n} f_{w}-\kappa_{g}^{1} \kappa_{g}^{2} f_{x}+\left(\left(\kappa_{g}^{1}\right)^{2}+\left(\kappa_{g}^{2}\right)^{2}\right) f_{y}+\left(\kappa_{g}^{2}\right)^{2} f_{z}\right), \\
\overline{\kappa_{g}^{1}} & =\frac{-1}{\nu_{23} \nu_{28}}\left(-\kappa_{g}^{1} \kappa_{g}^{2} \nu_{24}+\left(\left(\kappa_{g}^{1}\right)^{2}+\left(\kappa_{g}^{2}\right)^{2}\right) \nu_{25}+\left(\kappa_{g}^{2}\right)^{2} \nu_{26}+\kappa_{g}^{1} \kappa_{n} \nu_{27}\right), \\
\overline{\kappa_{g}^{2}} & =\frac{1}{\nu_{28}^{2} \nu_{33}}\left(\begin{array}{c}
\nu_{28}\left(-\kappa_{n} \nu_{27} \nu_{29}+\nu_{25}\left(-\kappa_{g}^{1} \nu_{29}+\kappa_{g}^{2} \nu_{31}\right)+\nu_{29} \nu_{24}^{\prime}+\right. \\
\left.\nu_{30}\left(\kappa_{g}^{1} \nu_{24}-\kappa_{g}^{2} \nu_{26}+\nu_{25}^{\prime}\right)+\nu_{31} \nu_{26}^{\prime}+\nu_{32}\left(\kappa_{n} \nu_{24}+\nu_{27}^{\prime}\right)\right)- \\
\left(\nu_{24} \nu_{29}+\nu_{25} \nu_{30}+\nu_{26} \nu_{31}+\nu_{27} \nu_{32}\right) \nu_{28}^{\prime}
\end{array}\right), \\
\overline{\tau_{g}^{1}} & =\frac{1}{\nu_{28}^{2}\|\nabla f\|}\left(\begin{array}{c}
-f_{x}\left(\nu_{28}\left(\kappa_{g}^{1} \nu_{25}+\kappa_{n} \nu_{27}-\nu_{24}^{\prime}\right)+\nu_{24} \nu_{28}^{\prime}\right)+f_{y}\left(\nu _ { 2 8 } \left(\kappa_{g}^{1} \nu_{24}-\right.\right. \\
\left.\left.\kappa_{26}+\nu_{25}^{\prime}\right)-\nu_{25} \nu_{28}^{\prime}\right)+f_{z}\left(\nu_{28}\left(\kappa_{g}^{2} \nu_{25}+\nu_{26}^{\prime}\right)-\nu_{26} \nu_{28}^{\prime}\right)+ \\
f_{w}\left(\nu_{28}\left(\kappa_{n} \nu_{24}+\nu_{27}^{\prime}\right)-\nu_{27} \nu_{28}^{\prime}\right)
\end{array}\right), \\
\overline{\tau_{g}^{2}}= & \frac{1}{\nu_{33}^{2}\|\nabla f\|}\left(\begin{array}{c}
-f_{x}\left(\nu_{33}\left(\kappa_{g}^{1} \nu_{30}+\kappa_{n} \nu_{32}-\nu_{29}^{\prime}\right)+\nu_{29} \nu_{33}\right)+f_{y}\left(\nu _ { 3 3 } \left(\kappa_{g}^{1} \nu_{29}-\right.\right. \\
\left.\left.\kappa_{g}^{2} \nu_{31}+\nu_{30}^{\prime}\right)-\nu_{30} \nu_{33}^{\prime}\right)+f_{z}\left(\nu_{33}\left(\kappa_{g}^{2} \nu_{30}+\nu_{31}^{\prime}\right)-\nu_{31} \nu_{33}^{\prime}\right)+ \\
f_{w}\left(\nu_{33}\left(\kappa_{n} \nu_{29}+\nu_{32}^{\prime}\right)-\nu_{32} \nu_{33}^{\prime}\right)
\end{array}\right),
\end{aligned}
$$

where

$$
\begin{aligned}
\nu_{23} & =\sqrt{2\left(\kappa_{g}^{2}\right)^{2}+\left(\kappa_{g}^{1}\right)^{2}}, \nu_{24}=-\sqrt{2} \kappa_{g}^{1} \kappa_{g}^{2}\|\nabla f\|+2 \sigma_{3} f_{x}, \\
\nu_{25} & =\sqrt{2}\left(\left(\kappa_{g}^{1}\right)^{2}+\left(\kappa_{g}^{2}\right)^{2}\right)\|\nabla f\|+2 \sigma_{3} f_{y}, \nu_{26}=\sqrt{2}\left(\kappa_{g}^{2}\right)^{2}\|\nabla f\|+2 \sigma_{3} f_{z}, \\
\nu_{27} & =\sqrt{2} \kappa_{g}^{1} \kappa_{n}\|\nabla f\|+2 \sigma_{3} f_{w}, \nu_{28}^{2}=\nu_{12}^{2}+\nu_{13}^{2}+\nu_{14}^{2}+\nu_{15}^{2}, \\
\nu_{29} & =\kappa_{g}^{2} \nu_{25} f_{w}+\kappa_{g}^{2} \nu_{26} f_{w}-\kappa_{g}^{2} \nu_{27} f_{y}-\kappa_{g}^{2} \nu_{27} f_{z}, \\
\nu_{19} & =-\kappa_{g}^{2} \nu_{24} f_{w}-\kappa_{g}^{1} \nu_{26} f_{w}+\kappa_{g}^{2} \nu_{27} f_{x}+\kappa_{g}^{1} \nu_{27} f_{z}, \\
\nu_{20} & =-\kappa_{g}^{2} \nu_{24} f_{w}+\kappa_{g}^{1} \nu_{25} f_{w}+\kappa_{g}^{2} \nu_{27} f_{x}-\kappa_{g}^{1} \nu_{27} f_{y}, \\
\nu_{21} & =\kappa_{g}^{2} \nu_{24} f_{y}+\nu_{26}\left(-\kappa_{g}^{2} f_{x}+\kappa_{g}^{1} f_{y}\right)+\kappa_{g}^{2} \nu_{24} f_{z}-\nu_{25}\left(\kappa_{g}^{2} f_{x}+\kappa_{g}^{1} f_{z}\right), \\
\nu_{22} & =\nu_{23} \nu_{28}\|\nabla f\| .
\end{aligned}
$$




\subsection{PN-Smarandache curves.}

Definition 3.5. Let $M$ be an oriented hypersurface in $\mathbb{E}^{4}$ and the Frenet curve $\delta=\delta(s)$ lying fully on $M$ with Darboux frame $\{\mathbf{T}, \mathbf{P}, \mathbf{U}, \mathbf{N}\}$ and non-zero curvatures $\kappa_{n}, \kappa_{g}^{1}, \kappa_{g}^{2}, \tau_{g}^{1}$ and $\tau_{g}^{2}$. Then the $\mathbf{P N}$-Smarandache curve of $\delta$ is defined as

$$
\delta(u)=\frac{1}{\sqrt{2}}(\mathbf{P}+\mathbf{N}) .
$$

Theorem 3.4. Let $r=r(u)$ be a Frenet curve lying on a hypersurface $M$ in $\mathbb{E}^{4}$ with Darboux frame $\{\mathbf{T}, \mathbf{P}, \mathbf{U}, \mathbf{N}\}$ and non-zero curvatures $\kappa_{n}, \kappa_{g}^{1}, \kappa_{g}^{2}, \tau_{g}^{1}$ and $\tau_{g}^{2}$. Then the curvature functions of the $\mathbf{P N}-$ Smarandache curve of $r$ satisfy the following equations:

$$
\begin{aligned}
& \overline{\kappa_{n}}=\frac{-1}{\xi_{1}\|\nabla f\|}\left(\begin{array}{c}
\left(\kappa_{n}\left(\kappa_{g}^{1}+\kappa_{n}\right)+\left(\tau_{g}^{1}\right)^{2}+\tau_{g}^{2}\left(-\kappa_{g}^{2}+\tau_{g}^{2}\right)\right) f_{w}+\left(-\kappa_{g}^{1}+\kappa_{n}\right) \tau_{g}^{1} f_{x} \\
+\left(\left(\kappa_{g}^{2}\right)^{2}+\kappa_{g}^{1}\left(\kappa_{g}^{1}+\kappa_{n}\right)+\left(\tau_{g}^{1}\right)^{2}-\kappa_{g}^{2} \tau_{g}^{2}\right) f_{y}+\tau_{g}^{1}\left(\kappa_{g}^{2}+\tau_{g}^{2}\right) f_{z}
\end{array}\right), \\
& \overline{\kappa_{g}^{1}}=\frac{-1}{\xi_{1} \xi_{6}}\left(\begin{array}{c}
\left(\kappa_{n}\left(\kappa_{g}^{1}+\kappa_{n}\right)+\left(\tau_{g}^{1}\right)^{2}+\tau_{g}^{2}\left(-\kappa_{g}^{2}+\tau_{g}^{2}\right)\right) \xi_{5}+\left(-\kappa_{g}^{1}+\kappa_{n}\right) \tau_{g}^{1} \xi_{2}+ \\
\left(\left(\kappa_{g}^{2}\right)^{2}+\kappa_{g}^{1}\left(\kappa_{g}^{1}+\kappa_{n}\right)+\left(\tau_{g}^{1}\right)^{2}-\kappa_{g}^{2} \tau_{g}^{2}\right) \xi_{3}+\tau_{g}^{1}\left(\kappa_{g}^{2}+\tau_{g}^{2}\right) \xi_{4}
\end{array}\right), \\
& \overline{\kappa_{g}^{2}}=\frac{1}{\xi_{6}^{2} \xi_{11}}\left(\begin{array}{c}
-\left(\xi_{2} \xi_{7}+\xi_{3} \xi_{8}+\xi_{4} \xi_{9}+\xi_{5} \xi_{10}\right) \xi_{6}^{\prime}+\xi_{6}\left(\left(\kappa_{g}^{1} \xi_{2}-\kappa_{g}^{2} \xi_{4}\right) \xi_{8}\right. \\
-\xi_{5}\left(\kappa_{n} \xi_{7}+\tau_{g}^{1} \xi_{8}+\tau_{g}^{2} \xi_{9}\right)+\xi_{3}\left(-\kappa_{g}^{1} \xi_{7}+\kappa_{g}^{2} \xi_{9}+\tau_{g}^{1} \xi_{10}\right)+ \\
\left.\xi_{7} \xi_{2}^{\prime}+\xi_{8} \xi_{3}^{\prime}+\xi_{9} \xi_{4}^{\prime}+\xi_{10}\left(\kappa_{n} \xi_{2}+\tau_{g}^{2} \xi_{4}+\xi_{5}^{\prime}\right)\right)
\end{array}\right), \\
& \overline{\tau_{g}^{1}}=\frac{1}{\xi_{6}^{2}\|\nabla f\|}\left(\begin{array}{c}
-\left(f_{x} \xi_{2}+f_{y} \xi_{3}+f_{z} \xi_{4}+f_{w} \xi_{5}\right) \xi_{6}^{\prime}+\xi_{6}\left(-\kappa_{g}^{1} f_{x} \xi_{3}+\kappa_{g}^{2} f_{z} \xi_{3}\right. \\
-\kappa_{n} f_{x} \xi_{5}-\tau_{g}^{2} f_{z} \xi_{5}+f_{x} \xi_{2}^{\prime}+f_{y}\left(\kappa_{g}^{1} \xi_{2}-\kappa_{g}^{2} \xi_{4}-\tau_{g}^{1} \xi_{5}\right. \\
\left.\left.+\xi_{3}^{\prime}\right)+f_{z} \xi_{4}^{\prime}+f_{w}\left(\kappa_{n} \xi_{2}+\tau_{g}^{1} \xi_{3}+\tau_{g}^{2} \xi_{4}+\xi_{5}^{\prime}\right)\right) \\
-\left(f_{x} \xi_{7}+f_{y} \xi_{8}+f_{z} \xi_{9}+f_{w} \xi_{10}\right) \xi_{11}^{\prime}+\xi_{11}\left(-\kappa_{g}^{1} f_{x} \xi_{8}+\right. \\
\kappa_{g}^{2} f_{z} \xi_{8}-\kappa_{n} f_{x} \xi_{10}-\tau_{g}^{2} f_{z} \xi_{10}+f_{x} \xi_{7}^{\prime}+f_{y}\left(\kappa_{g}^{1} \xi_{7}-\right. \\
\xi_{11}^{2}\|\nabla f\|
\end{array}\left(\begin{array}{c}
1 \\
\left.\left.\kappa_{g}^{2} \xi_{9}-\tau_{g}^{1} \xi_{10}+\xi_{8}^{\prime}\right)+f_{z} \xi_{9}^{\prime}+f_{w}\left(\kappa_{n} \xi_{7}+\tau_{g}^{1} \xi_{8}+\tau_{g}^{2} \xi_{9}+\xi_{10}^{\prime}\right)\right)
\end{array}\right),\right.
\end{aligned}
$$

Proof. Let $\delta=\delta(s)$ be a $\mathbf{P N}-$ Smarandache curve reference to Frenet curve $r$. Then, by differentiating Eq. (3.11), we obtain

$$
\begin{aligned}
\delta^{\prime}= & \frac{-\left(\kappa_{g}^{1}+\kappa_{n}\right) \mathbf{T}-\tau_{g}^{1} \mathbf{P}+\left(\kappa_{g}^{2}-\tau_{g}^{2}\right) \mathbf{U}+\tau_{g}^{1} \mathbf{N}}{\sqrt{2}}, \\
\delta^{\prime \prime}= & \frac{1}{\sqrt{2}}\left(\kappa_{g}^{1}-\kappa_{n}\right) \tau_{g}^{1} \mathbf{T}+\left(-\left(\kappa_{g}^{2}\right)^{2}-\kappa_{g}^{1}\left(\kappa_{g}^{1}+\kappa_{n}\right)-\left(\tau_{g}^{1}\right)^{2}+\kappa_{g}^{2} \tau_{g}^{2}\right) \mathbf{P} \\
& -\tau_{g}^{1}\left(\kappa_{g}^{2}+\tau_{g}^{2}\right) \mathbf{U}+\left(-\kappa_{n}\left(\kappa_{g}^{1}+\kappa_{n}\right)-\left(\tau_{g}^{1}\right)^{2}+\kappa_{g}^{2} \tau_{g}^{2}-\left(\tau_{g}^{2}\right)^{2}\right) \mathbf{N} .
\end{aligned}
$$

Therefore, from Eqs. (3.13), we get

$$
\begin{aligned}
& \overline{\mathbf{T}}=\frac{-\left(\kappa_{g}^{1}+\kappa_{n}\right) \mathbf{T}-\tau_{g}^{1} \mathbf{P}+\left(\kappa_{g}^{2}-\tau_{g}^{2}\right) \mathbf{U}+\tau_{g}^{1} \mathbf{N}}{\xi_{1}}, \\
& \overline{\mathbf{N}}=\frac{f_{x} \mathbf{T}+f_{y} \mathbf{P}+f_{z} \mathbf{U}+f_{w} \mathbf{N}}{\|\nabla f\|},
\end{aligned}
$$


where

$$
\xi_{1}=\sqrt{2\left(\tau_{g}^{1}\right)^{2}+\left(\kappa_{g}^{1}+\kappa_{n}\right)^{2}+\left(\kappa_{g}^{2}-\tau_{g}^{2}\right)^{2}} .
$$

On the other hand, we obtain

$$
\bar{P}=\frac{\xi_{2} \mathbf{T}+\xi_{3} \mathbf{P}+\xi_{4} \mathbf{U}+\xi_{5} \mathbf{N}}{\xi_{6}}
$$

where

$$
\begin{aligned}
& \xi_{2}=\sqrt{2}\left(-\kappa_{g}^{1}+\kappa_{n}\right) \tau_{g}^{1}\|\nabla f\|+2 \sigma_{4} f_{x} \\
& \xi_{3}=\sqrt{2}\left(\left(\kappa_{g}^{2}\right)^{2}+\kappa_{g}^{1}\left(\kappa_{g}^{1}+\kappa_{n}\right)+\left(\tau_{g}^{1}\right)^{2}-\kappa_{g}^{2} \tau_{g}^{2}\right)\|\nabla f\|+2 \sigma_{4} f_{y} \\
& \xi_{4}=\sqrt{2} \tau_{g}^{1}\left(\kappa_{g}^{2}+\tau_{g}^{2}\right)\|\nabla f\|+2 \sigma_{4} f_{z} \\
& \xi_{5}=\sqrt{2}\left(\kappa_{n}\left(\kappa_{g}^{1}+\kappa_{n}\right)+\left(\tau_{g}^{1}\right)^{2}-\kappa_{g}^{2} \tau_{g}^{2}+\left(\tau_{g}^{2}\right)^{2}\right)\|\nabla f\|+2 \sigma_{4} f_{w} \\
& \xi_{6}^{2}=\xi_{2}^{2}+\xi_{3}^{2}+\xi_{4}^{2}+\xi_{5}^{2}, \\
& \sigma_{4}=\left\langle\delta^{\prime \prime}, \mathbf{N}\right\rangle=\frac{1}{2\|\nabla f\|}\left(\begin{array}{c}
-\left(\kappa_{n}\left(\kappa_{g}^{1}+\kappa_{n}\right)+\left(\tau_{g}^{1}\right)^{2}+\tau_{g}^{2}\left(-\kappa_{g}^{2}+\tau_{g}^{2}\right)\right) f_{w}+ \\
\left(\kappa_{g}^{1}-\kappa_{n}\right) \tau_{g}^{1} f_{x}-\left(\left(\kappa_{g}^{2}\right)^{2}+\kappa_{g}^{1}\left(\kappa_{g}^{1}+\kappa_{n}\right)+\right. \\
\left.\left(\tau_{g}^{1}\right)^{2}-\kappa_{g}^{2} \tau_{g}^{2}\right) f_{y}-\tau_{g}^{1}\left(\kappa_{g}^{2}+\tau_{g}^{2}\right) f_{z}
\end{array}\right)
\end{aligned}
$$

Also, we have

$$
\bar{U}=\frac{\xi_{7} \mathbf{T}+\xi_{8} \mathbf{P}+\xi_{9} \mathbf{U}+\xi_{10} \mathbf{N}}{\xi_{11}}
$$

where,

$$
\begin{aligned}
\xi_{7}= & \kappa_{g}^{2} f_{w} \xi_{3}-\tau_{g}^{2} f_{w} \xi_{3}-\tau_{g}^{1} f_{z} \xi_{3}+\tau_{g}^{1} f_{w} \xi_{4}+\tau_{g}^{1} f_{y} \xi_{4}+\left(-\left(\kappa_{g}^{2}-\tau_{g}^{2}\right) f_{y}-\tau_{g}^{1} f_{z}\right) \xi_{5}, \\
\xi_{8}= & f_{w}\left(\left(-\kappa_{g}^{2}+\tau_{g}^{2}\right) \xi_{2}-\left(\kappa_{g}^{1}+\kappa_{n}\right) \xi_{4}\right)+f_{z}\left(\tau_{g}^{1} \xi_{2}+\left(\kappa_{g}^{1}+\kappa_{n}\right) \xi_{5}\right)-f_{x}\left(\tau_{g}^{1} \xi_{4}+\left(-\kappa_{g}^{2}+\tau_{g}^{2}\right) \xi_{5}\right), \\
\xi_{9}= & f_{w}\left(-\tau_{g}^{1} \xi_{2}+\left(\kappa_{g}^{1}+\kappa_{n}\right) \xi_{3}\right)+\tau_{g}^{1} f_{x}\left(\xi_{3}+\xi_{5}\right)-f_{y}\left(\tau_{g}^{1} \xi_{2}+\left(\kappa_{g}^{1}+\kappa_{n}\right) \xi_{5}\right), \\
\xi_{10}= & \left(\kappa_{g}^{2} f_{y} \xi_{2}-\tau_{g}^{2} f_{y} \xi_{2}+\tau_{g}^{1} f_{z} \xi_{2}-\kappa_{g}^{2} f_{x} \xi_{3}+\tau_{g}^{2} f_{x} \xi_{3}-\kappa_{g}^{1} f_{z} \xi_{3}-\kappa_{n} f_{z} \xi_{3}-\right. \\
& \left.\tau_{g}^{1} f_{x} \xi_{4}+\kappa_{g}^{1} f_{y} \xi_{4}+\kappa_{n} f_{y} \xi_{4}\right), \\
\xi_{11}= & \xi_{1}\|\nabla f\| \xi_{6} .
\end{aligned}
$$

In the light of the above calculations, the curvature functions $\overline{\kappa_{n}}, \overline{\kappa_{g}^{1}}, \overline{\kappa_{g}^{2}}, \overline{\tau_{g}^{1}}$ and $\overline{\tau_{g}^{2}}$ of $\delta$ are computed as in Eqs. (3.12). 
Corollary 3.7. If $r$ be asymptotic curve. Then, the following equations hold:

$$
\begin{aligned}
\overline{\mathbf{T}} & =\frac{-\kappa_{g}^{1} \mathbf{T}-\tau_{g}^{1} \mathbf{P}+\left(\kappa_{g}^{2}-\tau_{g}^{2}\right) \mathbf{U}+\tau_{g}^{1} \mathbf{N}}{\xi_{12}}, \\
\overline{\mathbf{N}} & =\frac{f_{x} \mathbf{T}+f_{y} \mathbf{P}+f_{z} \mathbf{U}+f_{w} \mathbf{N}}{\|\nabla f\|}, \\
\overline{\mathbf{P}} & =\frac{\xi_{13} \mathbf{T}+\xi_{14} \mathbf{P}+\xi_{15} \mathbf{U}+\xi_{16} \mathbf{N}}{\xi_{17}} \\
\overline{\mathbf{U}} & =\frac{\xi_{18} \mathbf{T}+\xi_{19} \mathbf{P}+\xi_{20} \mathbf{U}+\xi_{21} \mathbf{N}}{\xi_{22}}
\end{aligned}
$$

Thus, the curvature functions can be computed as follows:

$$
\begin{aligned}
& \overline{\kappa_{n}}=\frac{-1}{\|\nabla f\| \xi_{12}}\left(\begin{array}{c}
\left(\left(\tau_{g}^{1}\right)^{2}+\tau_{g}^{2}\left(-\kappa_{g}^{2}+\tau_{g}^{2}\right)\right) f_{w}-\kappa_{g}^{1} \tau_{g}^{1} f_{x}+\left(\left(\kappa_{g}^{1}\right)^{2}+\left(\kappa_{g}^{2}\right)^{2}+\right. \\
\left.\left(\tau_{g}^{1}\right)^{2}-\kappa_{g}^{2} \tau_{g}^{2}\right) f_{y}+\tau_{g}^{1}\left(\kappa_{g}^{2}+\tau_{g}^{2}\right) f_{z}
\end{array}\right), \\
& \overline{\kappa_{g}^{1}}=\frac{-1}{\xi_{12} \xi_{17}}\left(\begin{array}{c}
\left(\left(\tau_{g}^{1}\right)^{2}+\tau_{g}^{2}\left(-\kappa_{g}^{2}+\tau_{g}^{2}\right)\right) \xi_{16}-\kappa_{g}^{1} \tau_{g}^{1} \xi_{13}+\left(\left(\kappa_{g}^{1}\right)^{2}+\right. \\
\left.\left(\kappa_{g}^{2}\right)^{2}+\left(\tau_{g}^{1}\right)^{2}-\kappa_{g}^{2} \tau_{g}^{2}\right) \xi_{14}+\tau_{g}^{1}\left(\kappa_{g}^{2}+\tau_{g}^{2}\right) \xi_{15}
\end{array}\right), \\
& \overline{\kappa_{g}^{2}}=\frac{1}{\xi_{17}^{2} \xi_{22}}\left(\begin{array}{c}
\xi_{17}\left(\kappa_{g}^{1} \xi_{13} \xi_{19}-\kappa_{g}^{2} \xi_{15} \xi_{19}-\tau_{g}^{1} \xi_{16} \xi_{19}-\tau_{g}^{2} \xi_{16} \xi_{20}+\right. \\
\tau_{g}^{2} \xi_{15} \xi_{21}+\xi_{14}\left(-\kappa_{g}^{1} \xi_{18}+\kappa_{g}^{2} \xi_{20}+\tau_{g}^{1} \xi_{21}\right)+\xi_{18} \xi_{13}^{\prime}+\xi_{19} \xi_{14}^{\prime}+ \\
\left.\xi_{20} \xi_{15}^{\prime}+\xi_{21} \xi_{16}^{\prime}\right)-\left(\xi_{13} \xi_{18}+\xi_{14} \xi_{19}+\xi_{15} \xi_{20}+\xi_{16} \xi_{21}\right) \xi_{17}^{\prime}
\end{array}\right), \\
& \overline{\tau_{g}^{1}}=\frac{1}{\xi_{17}^{2}\|\nabla f\|}\left(\begin{array}{c}
-f_{x}\left(\kappa_{g}^{1} \xi_{14} \xi_{17}-\xi_{17} \xi_{13}^{\prime}+\xi_{13} \xi_{17}^{\prime}\right)+f_{y}\left(\xi _ { 1 7 } \left(\kappa_{g}^{1} \xi_{13}-\kappa_{g}^{2} \xi_{15}-\right.\right. \\
\left.\left.\tau_{g}^{1} \xi_{16}+\xi_{14}^{\prime}\right)-\xi_{14} \xi_{17}^{\prime}\right)+f_{z}\left(\xi_{17}\left(\kappa_{g}^{2} \xi_{14}-\tau_{g}^{2} \xi_{16}+\xi_{15}^{\prime}\right)-\right. \\
\left.\xi_{15} \xi_{17}^{\prime}\right)+f_{w}\left(\xi_{17}\left(\tau_{g}^{1} \xi_{14}+\tau_{g}^{2} \xi_{15}+\xi_{16}^{\prime}\right)-\xi_{16} \xi_{17}^{\prime}\right)
\end{array}\right), \\
& \overline{\tau_{g}^{2}}=\frac{1}{\xi_{22}^{2}\|\nabla f\|}\left(\begin{array}{c}
-f_{x}\left(\kappa_{g}^{1} \xi_{19} \xi_{22}-\xi_{22} \xi_{18}^{\prime}+\xi_{18} \xi_{22}^{\prime}\right)+f_{y}\left(\xi _ { 2 2 } \left(\kappa_{g}^{1} \xi_{18}-\kappa_{g}^{2} \xi_{20}-\right.\right. \\
\left.\left.\tau_{g}^{1} \xi_{21}+\xi_{19}^{\prime}\right)-\xi_{19} \xi_{22}^{\prime}\right)+f_{z}\left(\xi_{22}\left(\kappa_{g}^{2} \xi_{19}-\tau_{g}^{2} \xi_{21}+\xi_{20}^{\prime}\right)-\right. \\
\left.\xi_{20} \xi_{22}^{\prime}\right)+f_{w}\left(\xi_{22}\left(\tau_{g}^{1} \xi_{19}+\tau_{g}^{2} \xi_{20}+\xi_{21}^{\prime}\right)-\xi_{21} \xi_{22}^{\prime}\right)
\end{array}\right),
\end{aligned}
$$

where

$$
\begin{aligned}
& \xi_{12}=\sqrt{2\left(\tau_{g}^{1}\right)^{2}+\left(\kappa_{g}^{1}\right)^{2}+\left(\kappa_{g}^{2}-\tau_{g}^{2}\right)^{2}}, \xi_{13}=\sqrt{2}\left(-\kappa_{g}^{1}\right) \tau_{g}^{1}\|\nabla f\|+2 \sigma_{4} f_{x}, \\
& \xi_{14}=\sqrt{2}\left(\left(\kappa_{g}^{2}\right)^{2}+\kappa_{g}^{1}\left(\kappa_{g}^{1}\right)+\left(\tau_{g}^{1}\right)^{2}-\kappa_{g}^{2} \tau_{g}^{2}\right)\|\nabla f\|+2 \sigma_{4} f_{y}, \\
& \xi_{15}=\sqrt{2} \tau_{g}^{1}\left(\kappa_{g}^{2}+\tau_{g}^{2}\right)\|\nabla f\|+2 \sigma_{4} f_{z}, \\
& \xi_{16}=\sqrt{2}\left(\left(\tau_{g}^{1}\right)^{2}-\kappa_{g}^{2} \tau_{g}^{2}+\left(\tau_{g}^{2}\right)^{2}\right)\|\nabla f\|+2 \sigma_{4} f_{w}, \xi_{17}^{2}=\xi_{2}^{2}+\xi_{3}^{2}+\xi_{4}^{2}+\xi_{5}^{2}, \\
& \xi_{18}=\kappa_{g}^{2} f_{w} \xi_{14}-\tau_{g}^{2} f_{w} \xi_{14}-\tau_{g}^{1} f_{z} \xi_{14}+\tau_{g}^{1} f_{w} \xi_{15}+\tau_{g}^{1} f_{y} \xi_{15}+\left(-\left(\kappa_{g}^{2}-\tau_{g}^{2}\right) f_{y}-\tau_{g}^{1} f_{z}\right) \xi_{16}, \\
& \xi_{19}=f_{w}\left(\left(-\kappa_{g}^{2}+\tau_{g}^{2}\right) \xi_{14}-\left(\kappa_{g}^{1}\right) \xi_{15}\right)+f_{z}\left(\tau_{g}^{1} \xi_{14}+\left(\kappa_{g}^{1}\right) \xi_{16}\right)-f_{x}\left(\tau_{g}^{1} \xi_{15}+\left(-\kappa_{g}^{2}+\tau_{g}^{2}\right) \xi_{16}\right), \\
& \xi_{20}=f_{w}\left(-\tau_{g}^{1} \xi_{14}+\left(\kappa_{g}^{1}\right) \xi_{14}\right)+\tau_{g}^{1} f_{x}\left(\xi_{14}+\xi_{16}\right)-f_{y}\left(\tau_{g}^{1} \xi_{14}+\left(\kappa_{g}^{1}\right) \xi_{16}\right), \\
& \xi_{21}=\kappa_{g}^{2} f_{y} \xi_{13}-\tau_{g}^{2} f_{y} \xi_{13}+\tau_{g}^{1} f_{z} \xi_{13}-\kappa_{g}^{2} f_{x} \xi_{14}+\tau_{g}^{2} f_{x} \xi_{14}-\kappa_{g}^{1} f_{z} \xi_{14}-\tau_{g}^{1} f_{x} \xi_{15}+\kappa_{g}^{1} f_{y} \xi_{15}, \\
& \xi_{22}=\xi_{12}\|\nabla f\| \xi_{17} .
\end{aligned}
$$


Corollary 3.8. If $r$ be line of curvature. Then, the following equations hold:

$$
\begin{aligned}
\overline{\mathbf{T}} & =\frac{-\left(\kappa_{g}^{1}+\kappa_{n}\right) \mathbf{T}+k_{g}^{2} \mathbf{U}}{\xi_{23}}, \\
\overline{\mathbf{N}} & =\frac{f_{x} \mathbf{T}+f_{y} \mathbf{P}+f_{z} \mathbf{U}+f_{w} \mathbf{N}}{\|\nabla f\|}, \\
\overline{\mathbf{P}} & =\frac{\xi_{24} \mathbf{T}+\xi_{25} \mathbf{P}+\xi_{26} \mathbf{U}+\xi_{27} \mathbf{N}}{\xi_{28}}, \\
\overline{\mathbf{U}} & =\frac{\xi_{29} \mathbf{T}+\xi_{30} \mathbf{P}+\xi_{31} \mathbf{U}+\xi_{32} \mathbf{N}}{\xi_{33}} .
\end{aligned}
$$

Thus, the curvature functions can be computed as follows:

$$
\begin{aligned}
\overline{\kappa_{n}} & =-\frac{\kappa_{n}\left(\kappa_{g}^{1}+\kappa_{n}\right) f_{w}+\left(\left(\kappa_{g}^{2}\right)^{2}+\kappa_{g}^{1}\left(\kappa_{g}^{1}+\kappa_{n}\right)\right) f_{y}}{\|\nabla f\| \xi_{23}}, \\
\overline{\kappa_{g}^{1}} & =-\frac{\kappa_{n}\left(\kappa_{g}^{1}+\kappa_{n}\right) \xi_{25}+\left(\left(\kappa_{g}^{2}\right)^{2}+\kappa_{g}^{1}\left(\kappa_{g}^{1}+\kappa_{n}\right)\right) \xi_{27}}{\xi_{23} \xi_{28}}, \\
\overline{\kappa_{g}^{2}} & =\frac{1}{\xi_{28}^{2} \xi_{33}}\left(\begin{array}{c}
\xi_{28}\left(-\kappa_{n} \xi_{27} \xi_{29}+\xi_{25}\left(-\kappa_{g}^{1} \xi_{29}+\kappa_{g}^{2} \xi_{31}\right)+\xi_{29} \xi_{24}^{\prime}+\right. \\
\xi_{30}\left(\kappa_{g}^{1} \xi_{24}-\kappa_{g}^{2} \xi_{26}+\xi_{25}^{\prime}\right)+\xi_{31} \xi_{26}^{\prime}+\xi_{32}\left(\kappa_{n} \xi_{24}+\right. \\
\left.\left.\xi_{27}^{\prime}\right)\right)-\left(\xi_{24} \xi_{29}+\xi_{25} \xi_{30}+\xi_{26} \xi_{31}+\xi_{27} \xi_{32}\right) \xi_{28}^{\prime}
\end{array}\right), \\
\overline{\tau_{g}^{1}} & =\frac{1}{\xi_{28}^{2}\|\nabla f\|}\left(\begin{array}{c}
-f_{x}\left(\xi_{28}\left(\kappa_{g}^{1} \xi_{25}+\kappa_{n} \xi_{27}-\xi_{24}^{\prime}\right)+\xi_{24} \xi_{28}^{\prime}\right)+f_{y}\left(\xi _ { 2 8 } \left(\kappa_{g}^{1} \xi_{24}-\right.\right. \\
\left.\left.\kappa_{g}^{2} \xi_{26}+\xi_{25}^{\prime}\right)-\xi_{25} \xi_{28}^{\prime}\right)+f_{z}\left(\xi_{28}\left(\kappa_{g}^{2} \xi_{25}+\xi_{26}^{\prime}\right)-\xi_{26} \xi_{28}^{\prime}\right)+ \\
f_{w}\left(\xi-28\left(\kappa_{n} \xi_{24}+\xi_{27}^{\prime}\right)-\xi_{27} \xi_{28}^{\prime}\right)
\end{array}\right), \\
\overline{\tau_{g}^{2}}= & \frac{1}{\xi_{33}^{2}\|\nabla f\|}\left(\begin{array}{c}
-f_{x}\left(\xi_{33}\left(\kappa_{g}^{1} \xi_{30}+\kappa_{n} \xi_{32}-\xi_{29}^{\prime}\right)+\xi_{29} \xi_{33}^{\prime}\right)+f_{y}\left(\xi _ { 3 3 } \left(\kappa_{g}^{1} \xi_{29}-\right.\right. \\
\left.\left.\kappa_{g}^{2} \xi_{31}+\xi_{30}^{\prime}\right)-\xi_{30} \xi_{33}^{\prime}\right)+f_{z}\left(\xi_{33}\left(\kappa_{g}^{2} \xi_{30}+\xi_{31}^{\prime}\right)-\xi_{31} \xi_{33}^{\prime}\right)+ \\
f_{w}\left(\xi-33\left(\kappa_{n} \xi_{29}+\xi_{32}^{\prime}\right)-\xi_{32} \xi_{33}^{\prime}\right)
\end{array}\right),
\end{aligned}
$$

where

$$
\begin{aligned}
& \xi_{23}=\sqrt{\left(\kappa_{g}^{1}+\kappa_{n}\right)^{2}-\left(\kappa_{g}^{2}\right)^{2}}, \xi_{24}=2 \sigma_{4} f_{x}, \\
& \xi_{25}=\sqrt{2}\left(\left(\kappa_{g}^{2}\right)^{2}+\kappa_{g}^{1}\left(\kappa_{g}^{1}+\kappa_{n}\right)\right)\|\nabla f\|+2 \sigma_{4} f_{y}, \xi_{26}=2 \sigma_{4} f_{z}, \\
& \xi_{27}=\sqrt{2} \kappa_{n}\left(\kappa_{g}^{1}+\kappa_{n}\right)\|\nabla f\|+2 \sigma_{4} f_{w}, \xi_{28}^{2}=\xi_{24}^{2}+\xi_{25}^{2}+\xi_{26}^{2}+\xi_{27}^{2}, \\
& \xi_{29}=\left(k g 2 \xi_{25} f_{w}-\kappa_{g}^{2} \xi_{27} f_{y}\right), \\
& \xi_{30}=-\kappa_{g}^{2} \xi_{24} f_{w}-\kappa_{g}^{1} \xi_{26} f_{w}-\kappa_{n} \xi_{26}[u] f_{w}+\kappa_{g}^{2} \xi_{27} f_{x}+\left(\kappa_{g}^{1}+\kappa_{n}\right) \xi_{27} f_{z}, \\
& \xi_{31}=\left(\kappa_{g}^{1}+\kappa_{n}\right) \xi_{25} f_{w}-\kappa_{g}^{1} \xi_{27} f_{y}-\kappa_{n} \xi_{27} f_{y}, \\
& \xi_{32}=\kappa_{g}^{2} \xi_{24} f_{y}+\kappa_{g}^{1} \xi_{26} f_{y}+\kappa_{n} \xi_{26} f_{y}-\xi_{25}\left(\kappa_{g}^{2} f_{x}+\left(\kappa_{g}^{1}+\kappa_{n}\right) f_{z}\right), \\
& \xi_{33}=\xi_{23}\|\nabla f\| \xi_{28} .
\end{aligned}
$$




\section{EXAMPLES}

Example 4.1. Consider the curve $r(u)$ given by

$$
r(u)=(\cos (u), \sin (u), \cos (2 u), \sin (2 u)) .
$$

By using the definition 2.6, we can calculate the Darboux frame $\{\mathbf{T}, \mathbf{P}, \mathbf{U}, \mathbf{N}\}$ as follows:

$$
\begin{gathered}
\mathbf{T}=\left(-\frac{\sin (u)}{5}, \frac{\cos (u)}{5},-\frac{2}{5} \sin (2 u), \frac{2}{5} \cos (2 u)\right), \\
\mathbf{N}=\left(\frac{\cos (u)}{\sqrt{2}}, \frac{\sin (u)}{\sqrt{2}}, \frac{\cos (2 u)}{\sqrt{2}}, \frac{\sin (2 u)}{\sqrt{2}}\right), \\
\mathbf{P}=\left(\frac{\cos (u)}{\sqrt{2}}, \frac{\sin (u)}{\sqrt{2}},-\frac{\cos (2 u)}{\sqrt{2}},-\frac{\sin (2 u)}{\sqrt{2}}\right), \\
\mathbf{U}=\left(\frac{2 \sin (u)}{\sqrt{5}},-\frac{2 \cos (u)}{\sqrt{5}},-\frac{1}{\sqrt{5}} \sin (2 u), \frac{1}{\sqrt{5}} \cos (2 u)\right) .
\end{gathered}
$$

The curvature functions of the curve $r$ can be computed as follows:

$$
\kappa_{n}=-\sqrt{\frac{5}{2}}, \kappa_{g}^{1}=\frac{3}{\sqrt{10}}, \kappa_{g}^{2}=-2 \sqrt{\frac{2}{5}}, \tau_{g}^{1}=\tau_{g}^{2}=0 .
$$

Therefore, we can obtain $\mathbf{T U}$-Smarndache curve as

$$
\phi=\frac{1}{\sqrt{2}}(\mathbf{T}+\mathbf{U})=\left(\frac{\sin (u)}{\sqrt{10}}, \frac{-\cos (u)}{\sqrt{10}}, \frac{-3 \sin (2 u)}{\sqrt{10}}, \frac{3 \cos (2 u)}{\sqrt{10}}\right) .
$$

By using the definition 2.6, we can obtain

$$
\begin{aligned}
\overline{\mathbf{T}} & =\frac{1}{\sqrt{37}}(\cos (u), \sin (u),-6 \cos (2 u),-6 \sin (2 u)), \\
\overline{\mathbf{N}} & =\frac{1}{2 \sqrt{5}}(\sin (u), \cos (u), 3 \sin (2 u), 3 \cos (2 u)), \\
\overline{\mathbf{P}} & =\frac{1}{\sqrt{16930}}(17 \sin (u),-17 \cos (u), 129 \sin (2 u),-129 \cos (2 u)), \\
\overline{\mathbf{U}} & =\frac{1}{\sqrt{8465}}(-54 \cos (u),-54 \sin (u),-9 \cos (2 u),-9 \sin (2 u)) .
\end{aligned}
$$

The curvature functions of the Smarandache curve $\phi$ can be computed as follows:

$$
\overline{\kappa_{n}}=\frac{-1}{2} \sqrt{\frac{37}{5}}, \quad \overline{\kappa_{g}^{1}}=\frac{1531}{\sqrt{626410}}, \quad \overline{\kappa_{g}^{2}}=\frac{-324 \sqrt{2}}{1693}, \quad \overline{\tau_{g}^{1}}=\overline{\tau_{g}^{2}}=0 .
$$

Example 4.2. Consider the unit speed curve given by

$$
r(u)=\left(\frac{\cos (u)}{2}, \frac{\sin (u)}{2}, \frac{u}{2}, \frac{u}{\sqrt{2}}\right) .
$$

By using the definition 2.6 we can calculate the Darboux frame $\{\mathbf{T}, \mathbf{P}, \mathbf{U}, \mathbf{N}\}$ as follow:

$$
\begin{aligned}
\mathbf{T} & =\left(\frac{-\sin (u)}{2}, \frac{\cos (u)}{2}, \frac{1}{2}, \frac{1}{\sqrt{2}}\right), \\
\mathbf{N} & =\left(\frac{\cos (u)}{2}, \frac{\sin (u)}{2}, \frac{1}{2}, \frac{1}{\sqrt{2}}\right),
\end{aligned}
$$




$$
\begin{aligned}
& \mathbf{P}=\left(\frac{-\sqrt{3}}{2} \cos (u), \frac{-\sqrt{3}}{2} \sin (u), \frac{1}{2 \sqrt{3}}, \frac{-1}{\sqrt{6}}\right), \\
& \mathbf{U}=\left(-\sqrt{\frac{2}{3}} \sin (u), \sqrt{\frac{2}{3}} \cos (u), \frac{-1}{\sqrt{6}},-\frac{1}{2 \sqrt{3}}\right),
\end{aligned}
$$

The curvature functions of the curve $r$ can be computed as follows

$$
\kappa_{n}=-\frac{1}{4}, \kappa_{g}^{1}=\frac{\sqrt{3}}{4}, \kappa_{g}^{2}=-\frac{1}{\sqrt{2}}, \tau_{g}^{1}=0, \tau_{g}^{2}=-\frac{1}{\sqrt{6}} .
$$

Therefore we can obtain $\mathbf{T N}$-Smarndache curve as

$$
\beta=\left(\frac{\cos (u)-\sin (u)}{2 \sqrt{2}}, \frac{\cos (u)+\sin (u)}{2 \sqrt{2}}, \frac{1}{\sqrt{2}}, 0\right) .
$$

By using the definition 2.6, we can obtain

$$
\begin{aligned}
& \overline{\mathbf{T}}=\left(-\frac{\cos (u)+\sin (u)}{2 \sqrt{2}}, \frac{\cos (u)-\sin (u)}{2 \sqrt{2}}, 0,0\right), \\
& \overline{\mathbf{N}}=\left(\frac{\cos (u)-\sin (u)}{2 \sqrt{2}}, \frac{\cos (u)+\sin (u)}{2 \sqrt{2}}, \frac{1}{2}, \frac{1}{2}\right), \\
& \overline{\mathbf{P}}=\left(\frac{-3(\cos (u)-\sin (u))}{\sqrt{22}}, \frac{3(\cos (u)+\sin (u))}{\sqrt{22}}, \frac{1}{\sqrt{11}}, \frac{1}{\sqrt{11}}\right), \\
& \overline{\mathbf{U}}=\left(0,0, \frac{1}{\sqrt{11}}, \frac{1}{\sqrt{11}}\right) .
\end{aligned}
$$

The curvature functions of the Smarandache curve $\beta$ can be computed as

$$
\overline{\kappa_{n}}=\frac{-1}{2}, \quad \overline{\kappa_{g}^{1}}=\frac{3}{\sqrt{11}}, \quad \overline{\kappa_{g}^{2}}=0, \quad \overline{\tau_{g}^{1}}=\overline{\tau_{g}^{2}}=0 .
$$

\section{Conclusion}

In the four dimensional Euclidean space $\mathbb{E}^{4}$, some special Smarandache curves lying on a hypersurface are investigated. Also, the differential geometric properties of these curves are obtained. Furthermore, some computational examples in support of our main results are given.

\section{ACKNOWLEDGMENT}

This research was supported by Islamic University of Madinah. We would like to thank our colleagues from Deanship of Scientific Research who provided insight and expertise that greatly assisted the research.

\section{REFERENCES}

[1] C. Ashbacher, Smarandache geometries, Smarandache Notions J. 8(1-3) (1997), 212-215.

[2] M. Khalifa Saad, Spacelike and timelike admissible Smarandache curves in pseudo-Galilean space, J. Egypt. Math. Soc. 24 (2016), 416-423.

[3] M. Çetin, H. Kocayiğit, On the quaternionic Smarandache curves in Euclidean 3- space, Int. J. Contemp. Math. Sci. 8(3) (2013), 139-150.

[4] M. Do Carmo, Differential Geometry of curves and surface, Englewood Cliffs, NJ, USA, Prentice Hall, 1976. 
[5] H.S. Abdel-Aziz, M. Khalifa Saad, Smarandache curves of some special curves in the Galilean 3-space, Honam Math. J. $37(2)$ (2015), 253-264.

[6] H.S. Abdelaziz, M. Khalifa Saad, Computation of Smarandache curves according to Darboux frame in Minkowski 3-space, J. Egypt. Math. Soc. 25 (2017), 382-390.

[7] H.S. Abdelaziz, M. Khalifa Saad, Some geometric invariants of pseudo-spherical evolutes in the hyperbolic 3-space, Comput. Mater. Continua 57(3) (2018), 389-415.

[8] A.T. Ali, Special Smarandache curves in the Euclidean space, Int. J. Math. Comb. 2 (2010), 30-36.

[9] O. Bektas, S. Yuce, Smarandache curves according to Darboux frame in Euclidean space, Rom. J. Math. Comput. Sci. 3(1) (2013), 48-59.

[10] M. Çetin, Y. Tunçer, M. K. Karacan, Smarandache curves according to Bishop frame in Euclidean space, Gen. Math. Notes 20(2) (2014), 50-66.

[11] M. Turgut, S. Yilmaz, Smarandache curves in Minkowski space-time, Int. J. Math. Comb. 3 (2008), 51-55.

[12] K. Taşk prü, M. Tosun, Smarandache curves according to Sabban frame on $\mathbb{S}^{2}$, Bol. Soc. Paran. Mat. 32(1) (2014), 51-59.

[13] O. Aléssio, Differential geometry of intersection curves in $\mathbb{R}^{4}$ of three implicit surfaces, Comput. Aided Geom. Des. 26 (2009), 455-471.

[14] H.S. Abdel-Aziz, M. Khalifa Saad, A. A. Abdel-Salam, On Implicit Surfaces and Their Intersection Curve in Euclidean 4-Space, Houston J. Math. 40(2) (2014), 339-352.

[15] B. O’Neill, Elementary Differential Geometry, Burlington, MA, USA, Academic Press, 1966.

[16] M. Düldü, B. Düldül, N. Kuruoğlu and E. Özdamar, Extension of the Darboux frame into Euclidean 4-space and its invariants, Turk. J. Math. 41 (2017), 1628-1639. 